\title{
Synthesis and Evaluation of Novel Pyrroles and Pyrrolopyrimidines as Anti-Hyperglycemic Agents
}

\author{
M. S. Mohamed, ${ }^{1}$ S. A. Ali, ${ }^{2}$ D. H. A. Abdelaziz, ${ }^{2}$ and Samar S. Fathallah ${ }^{1}$ \\ ${ }^{1}$ Pharmaceutical Organic Chemistry Department, Faculty of Pharmacy, Helwan University, Ein-Helwan, Helwan, Cairo 11795, Egypt \\ ${ }^{2}$ Biochemistry and Molecular Biology Department, Faculty of Pharmacy, Helwan University, Ein-Helwan, Helwan, Cairo 11795, Egypt
}

Correspondence should be addressed to Samar S. Fathallah; ssfathallah@yahoo.com

Received 12 February 2014; Revised 16 May 2014; Accepted 22 May 2014; Published 26 June 2014

Academic Editor: Paul M. Tulkens

Copyright (c) 2014 M. S. Mohamed et al. This is an open access article distributed under the Creative Commons Attribution License, which permits unrestricted use, distribution, and reproduction in any medium, provided the original work is properly cited.

\begin{abstract}
A series of pyrrole and pyrrolopyrimidine derivatives were examined for their in vivo antihyperglycemic activity. Compounds Ia-c,e, and IVg showed promising antihyperglycemic activity equivalent to a well-known standard antihyperglycemic drug, Glimepiride (Amaryl, $4 \mathrm{mg} / \mathrm{kg}$ ). In this paper, we examine and discuss the structure-activity relationships and antihyperglycemic activity of these compounds.
\end{abstract}

\section{Introduction}

For several decades, interest in pyrrole derivatives increases due to their pharmaceutical importance [1-3], such as antimicrobial [4-8], antiviral [9, 10], anti-inflammatory [11-13], analgesic [14], antitumor [15, 16], antihyperlipidemic [17], anticonvulsant [18], and antihyperglycemic agents $[19,20]$, as shown in Figures 1 and 2(a).

Likewise, the key roles played by purines and pyrimidines in cellular processes have made them valuable lead for drug discovery; among these, pyrrolo[3,2- $d$ ] pyrimidines, a class of 7-deazapurine analogs, exhibit interesting biological activity in part due to their resemblance to pyrimidines and purines. These huge therapeutic applications have motivated new efforts in the search for novel derivatives with improved biological activity and diverse applications in the pharmaceutical industry $[1-4,19,20]$.

Diabetes mellitus (DM) is a severe metabolic disorder that has a significant impact on the health and quality of patients' life. Treatment of diabetic patients has been focused on dietary management and oral antidiabetics, among these: sulfonylureas, metformin, acarbose, and others. However, some of the currently used antihyperglycemic have several adverse side effects like hepatotoxicity, weight gain, and hypoglycemia.
This situation emphasized the need to develop novel antihyperglycemic agents [21]. Glimepiride (Amaryl) is a sulfonylurea containing a pyrrole group, acting as antihyperglycemic drug [22]. It is sometimes classified either as the first third-generation sulfonylurea or as second-generation. Glimepiride is indicated to treat type 2 diabetes mellitus; its mode of action is to increase insulin production by the pancreas, as shown in Figure 2(b).

Recently, dipeptidyl peptidase IV (DPP-IV) inhibitors [23-25] have been shown to be effective and safe compounds that control blood glucose. Improvement of the inhibitory activity and chemical stability of a series of substituted piperidinyl glycine 2-cyano-4,5-methano pyrroline (DPPIV) inhibitors was, respectively, achieved by the introduction of pyrroline moiety at the 4 position and 1 position of the piperidinyl glycine, leading to a series of potent and stable DPP-IV inhibitors [25]. Two important DPP-IV inhibitors, having a pyrrole and fused pyrrole, vildaglipin, and saxagliptin $[24,25]$, are on the market in many countries, as shown in Figure 2(b).

A highly potent DPP-IV inhibitor thienopyrimidine was also reported [24]. While trying to maintain consistency of in vitro and in vivo biological activity, a simple scaffold replacement of thienopyrimidine with pyrrolopyrimidine lead to significantly improved metabolic stability [22-24], as shown in Figure 2(c). 


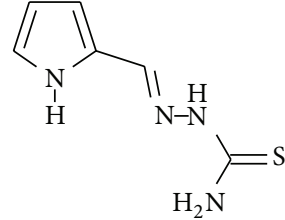

Pyrrole-2-carbaldehyde

Pyrrole as antibacterial and as HIV-1 integrase inhibition [4-8]

(a)<smiles>Cc1ccc(C(=O)c2ccc(CC(=O)O)n2C)cc1</smiles>

Pyrroles as nonsteroidal anti-inflammatory drugs (NSAIDs) [11-13]

(b)

Figure 1: Pyrrole as valuable leads in the drug discovery field.

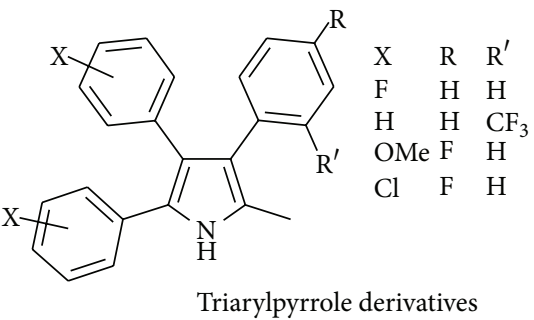

(a)<smiles>CCC1=C(C)CN(NC(=O)CCc2ccc(S(=O)(=O)NC(=O)NC3CCC(C)CC3)cc2)C1=O</smiles><smiles>N#C[C@H]1CCCN1C(=O)CNC12CC3CC(CC(O)(C3)C1)C2</smiles>

(b)<smiles>N#Cc1ccccc1Cn1c(N2CCC[C@@H](N)C2)nc2ccsc2c1=O</smiles>

Thienopyrimidines<smiles>[R16]C=[R15]C=[R]</smiles>

Pyrrolopyrimidines

(c)

FIgURe 2: (a) Pyrroles as antihyperglycemic agents [19, 20], (b) Amaryl, standard antihyperglycemic drug [21, 22], and approved DPP-IV inhibitors [23-25] as type 2 diabetes medications containing a pyrrole moiety. (c) Thieno and Pyrrolo-pyrimidines as DPP-IV inhibitors. 
Motivated by the importance of this system and in continuation of our research efforts [26-30], we try to highlight aspects reported on the chemistry of some newly synthesized pyrrole and pyrrolopyrimidine derivatives and evaluate them for the antihyperglycemic activities. The synthetic pathways adopted for the synthesis of these compounds are registered in Schemes 1-3.

\section{Materials and Methods}

2.1. Chemistry. All melting points were uncorrected and measured using Electrothermal IA 9100 apparatus (Shimadzu, Japan). IR spectra were recorded as potassium bromide pellets on a Perkin-Elmer 1650 spectrophotometer (USA), Faculty of Science, Cairo University, Cairo, Egypt. ${ }^{1} \mathrm{H}$ NMR and ${ }^{13} \mathrm{CNMR}$ spectra were performed on JOEL NMR FXQ$300 \mathrm{MHz}$ and JOELNMR FXQ-500 MHz spand chemical shifts were expressed as ppm against TMS as internal reference (Faculty of Science, Cairo University, Cairo, Egypt). Mass spectra were recorded at $70 \mathrm{eV}$ EI Ms-QP 1000 EX (Shimadzu, Japan), Faculty of Science, Cairo University, Cairo, Egypt. Microanalyses were operated using Vario, Elementar apparatus (Shimadzu, Japan), Organic Microanalysis Unit, Faculty of Science, Cairo University, Cairo, Egypt. Column chromatography was performed on (Merck) silica gel 60 (particle size $0.06-0.20 \mathrm{~mm}$ ). Compounds $\mathbf{I a}-\mathbf{c}, \mathbf{f}-\mathbf{h}, \mathbf{k}-\mathbf{m}$, IIa-c, f-h, IIIa-c, f-h, IVa-c, Va-c, VIa-c, VIIa-c,and VIIIa-c were prepared as reported in the literature [26-31]. All new compounds yielded spectral data consistent with the proposed structure and microanalysis within $\pm 0.4 \%$ of the theoretical values.

2.1.1. 2-Amino-1-(3,4-dichlorophenyl)-4,5-diphenyl-1H-pyrrole-3-carbonitrile Id (Scheme 1). A mixture of benzoin (2 g, $0.01 \mathrm{~mol}), 3,4$-dichlorophenyl amine $(1.6 \mathrm{~g}, 0.01 \mathrm{~mol})$ in dry benzene $(50 \mathrm{~mL})$, was kept at $80^{\circ} \mathrm{C}$ for $9 \mathrm{~h}$. The reaction mixture was cooled; then malononitrile $(0.66 \mathrm{mg}, 0.01 \mathrm{~mol})$ was added, followed by catalytic amount of pyridine $(2 \mathrm{~mL})$ portion wise and left to reflex till solid formed. The solvent was evaporated under reduced pressure and the residue was recrystallized from methanol to give Id . Yield: 45\%; M.P. $118-122^{\circ} \mathrm{C} ;{ }^{1} \mathrm{H}$ NMR (DMSO- $\left.d_{6}, 300 \mathrm{MHz}\right) \delta$ (ppm): 5.21 (br.s, $2 \mathrm{H}, \mathrm{NH}_{2}, \mathrm{D}_{2} \mathrm{O}$ exchangeable), 7.0-7.8 (m, 13H, Ar-H); ${ }^{13} \mathrm{C}$ NMR (DMSO- $d_{6}$ ): $\delta 114.33,118.24,119.37,125.8,126.18$, $127.80,128.45,129.84,130.29,132.16,132.70,133.62,134.96$, 142.05 ppm; IR (KBr) $v\left(\mathrm{~cm}^{-1}\right): 3410,3370\left(\mathrm{NH}_{2}\right), 2220$ $(\mathrm{C} \equiv \mathrm{N})$; MS (EI) $m / z: 403\left(\mathrm{M}^{+}, 14 \%\right), 405\left(\mathrm{M}^{+}+2,8.75 \%\right)$, $407\left(\mathrm{M}^{+}+4,1.1 \%\right)$; Anal. Calcd for $\mathrm{C}_{23} \mathrm{H}_{15} \mathrm{Cl}_{2} \mathrm{~N}_{3}$ (403.06): $\mathrm{C}$, 68.33; H, 3.74; Cl, 17.54; N, 10.39\%. Found: C, 68.55; H, 3.92; $\mathrm{Cl}, 17.23 ; \mathrm{N}, 10.72 \%$.

2.1.2. 2-Amino-1-(1,5-dimethyl-3-oxo-2-phenyl-2,3-dihydro-1Hpyrazol-4-yl)-4-phenyl-1H-pyrrole-3-carbonitrile Ie (Scheme 1). 1,5-Dimethyl-4-(2-oxo-2-phenylethylamino)-2-phenyl$1 H$-pyrazol-3 $(2 H)$-one $[27-30](3.22 \mathrm{~g}, 0.01 \mathrm{~mol})$ was dissolved in dry ethanol $(20 \mathrm{~mL})$; then malononitrile $(0.66 \mathrm{~g}$, $0.01 \mathrm{~mol})$ was added, followed by sodium ethoxide $(0.01 \mathrm{~mol})$ portion wise, and left to reflux till solid formed. The solvent was evaporated under reduced pressure and the residue was recrystallized from methanol to give Ie. Yield: 66\%; M.P. $163-166^{\circ} \mathrm{C} ;{ }^{1} \mathrm{H}$ NMR (DMSO- $\left.d_{6}, 300 \mathrm{MHz}\right) \delta$ (ppm): 2.43 (s, $3 \mathrm{H}, \mathrm{CH}_{3}$ ), 3.12 (s, 3H, N-CH $\mathrm{CH}_{3}$, 6.13 (br.s, $2 \mathrm{H}, \mathrm{NH}_{2}, \mathrm{D}_{2} \mathrm{O}$ exchangeable), 6.8-7.8 (m, $10 \mathrm{H}, \mathrm{Ar}-\mathrm{H}$ and $\left.1 \mathrm{H}, \mathrm{C}_{6}-\mathrm{H}\right)$; IR $(\mathrm{KBr}) v\left(\mathrm{~cm}^{-1}\right): 3410,3350\left(\mathrm{NH}_{2}\right), 2210(\mathrm{C} \equiv \mathrm{N}), 1703(\mathrm{C}=\mathrm{O})$; MS (EI) m/z: $369\left(\mathrm{M}^{+}, 23 \%\right), 370\left(\mathrm{M}^{+}+1,6.1 \%\right)$; Anal. Calcd for $\mathrm{C}_{22} \mathrm{H}_{19} \mathrm{~N}_{5} \mathrm{O}$ (369.16): C, 71.53; $\mathrm{H}, 5.18 ; \mathrm{N}, 18.96 ; \mathrm{O}, 4.33 \%$. Found: C, 71.55; H, 5.26; N, 18.70; O, 3.05\%.

2.1.3. 2-Ethoxymethylenamino-1,5-disubstituted-4-phenyl-1Hpyrrole-3-carbonitriles Ii,j (Scheme 1). Compound I, d, or e $(0.01 \mathrm{~mol})$ in triethyl orthoformate $(20 \mathrm{~mL})$ was refluxed for $9 \mathrm{~h}$. The solvent was removed under reduced pressure and the residue was recrystallized from methanol/water to give the target compounds $\mathbf{I i}, \mathbf{j}$.

2.1.4. Ethyl N-3-Cyano-1-(3,4-dichlorophenyl)-4,5-diphenyl1H-pyrrol-2-ylformimidate Ii. Yield: $60 \%$; M.P. $120-122^{\circ} \mathrm{C} ;{ }^{1} \mathrm{H}$ NMR (DMSO- $\left.d_{6}, 300 \mathrm{MHz}\right) \delta(\mathrm{ppm}): 1.30(\mathrm{t}, 3 \mathrm{H}, J=7.1 \mathrm{~Hz}$, $\left.\mathrm{CH}_{3}\right), 4.2\left(\mathrm{q}, 2 \mathrm{H}, \mathrm{J}=7.1 \mathrm{~Hz}, \mathrm{OCH}_{2}\right), 6.9-7.8(\mathrm{~m}, 14 \mathrm{H}, \mathrm{Ar}-\mathrm{H}$ and $\mathrm{N}=\mathrm{CH})$; IR $(\mathrm{KBr}) v\left(\mathrm{~cm}^{-1}\right): 3070,2900(\mathrm{CH}), 2310(\mathrm{CN})$, $1620(\mathrm{C}=\mathrm{C}), 1560(\mathrm{C}=\mathrm{N})$; MS (EI) $m / z: 460\left(\mathrm{M}^{+}, 13.5 \%\right)$, $462\left(\mathrm{M}^{+}+2,8.5 \%\right), 464\left(\mathrm{M}^{+}+4,2.71 \%\right)$; Anal. Calcd for $\mathrm{C}_{26} \mathrm{H}_{19} \mathrm{Cl}_{2} \mathrm{~N}_{3} \mathrm{O}$ (460.35): C, 67.83; H, 4.16; Cl, 15.40; N, 9.13; O, 3.48\%, Found: C, 68.03; H, 4.11; Cl, 15.63; N, 8.91; O, 3.69\%.

2.1.5. Ethyl N-3-Cyano-1-(1,5-dimethyl-3-oxo-2-phenyl-2,3dihydro-1H-pyrazol-4-yl)-4-phenyl-1H-pyrrol-2-ylacetimidate Ij. Yield: 56\%; M.P. $120-122^{\circ} \mathrm{C}$; ${ }^{1} \mathrm{H}$ NMR (DMSO- $d_{6}$, $300 \mathrm{MHz}) \delta(\mathrm{ppm}): 1.20\left(\mathrm{t}, 3 \mathrm{H}, J=7.2 \mathrm{~Hz}, \mathrm{CH}_{3}\right), 2.41(\mathrm{~s}, 3 \mathrm{H}$, $\left.\mathrm{CH}_{3}\right), 3.1\left(\mathrm{~s}, 3 \mathrm{H}, \mathrm{N}-\mathrm{CH}_{3}\right), 4.01\left(\mathrm{q}, 2 \mathrm{H}, J=7.1 \mathrm{~Hz}, \mathrm{OCH}_{2}\right)$, 6.9-7.8 (m, 12H, Ar-H, $\mathrm{C}_{6}-\mathrm{H}$ and $\left.\mathrm{N}=\mathrm{CH}\right) ; \mathrm{IR}(\mathrm{KBr}) v\left(\mathrm{~cm}^{-1}\right)$ : 3030, 2910(CH), 2240(CN), $1610(\mathrm{C}=\mathrm{C}), 1570(\mathrm{C}=\mathrm{N})$; MS (EI) $m / z: 425\left(\mathrm{M}^{+}, 6.5 \%\right), 426\left(\mathrm{M}^{+}+1,1.81 \%\right)$; Anal. Calcd for $\mathrm{C}_{26} \mathrm{H}_{23} \mathrm{~N}_{5} \mathrm{O}_{2}$ (425.19): C, 70.57; H, 5.45; N, 16.46; O, 7.52\%. Found: C, 70.21; H, 5.17; N, 16.12; O, 7.18\%.

2.1.6. N-(4-Phenyl-1,3,5-trisubstituted-1H-pyrrol-2-yl)-acetamides I n,o (Scheme 1). Compound I, d, or e (0.01 mol) in acetic anhydride $(30 \mathrm{~mL})$ was refluxed for $2 \mathrm{~h}$, cooled, poured onto ice water, neutralized with ammonia to give a precipitate which was filtered off, dried, and recrystallized from methanol to give the target compounds In, o.

2.1.7. N-(3-Cyano-1-(3,4-dichlorophenyl)-4,5-diphenyl-1Hpyrrol-2-yl) acetamide In. Yield: $66 \%$; M.P. $135-138^{\circ} \mathrm{C} ;{ }^{1} \mathrm{H}$ $\mathrm{NMR}\left(\mathrm{DMSO}-d_{6}, 300 \mathrm{MHz}\right) \delta(\mathrm{ppm}): 2.23\left(\mathrm{~s}, 3 \mathrm{H}, \mathrm{CO}-\mathrm{CH}_{3}\right)$, 7.0-7.8 (m, 13H, Ar- $\mathrm{H}), 9.5$ (s, 1H, NH, $\mathrm{D}_{2} \mathrm{O}$ exchangeable); IR $(\mathrm{KBr}) v\left(\mathrm{~cm}^{-1}\right): 3430(\mathrm{NH}), 2330(\mathrm{C} \equiv \mathrm{N}), 1710(\mathrm{C}=\mathrm{O}) ; \mathrm{MS}$ (EI) $m / z: 445\left(\mathrm{M}^{+}, 13.1 \%\right), 447\left(\mathrm{M}^{+}+2,7.9 \%\right), 449\left(\mathrm{M}^{+}+4\right.$, 0.91\%); Anal. Calcd for $\mathrm{C}_{25} \mathrm{H}_{17} \mathrm{Cl}_{2} \mathrm{~N}_{3} \mathrm{O}$ (445.07): C, 67.28; $\mathrm{H}$, 3.84; Cl, 15.89; N, 9.41; O, 3.58\%. Found: C, 67.47; H, 4.06; Cl, $16.22 ; \mathrm{N}, 9.57 ; \mathrm{O}, 3.66 \%$.

2.1.8. N-(3-Cyano-1-(1,5-dimethyl-3-oxo-2-phenyl-2,3-dihydro-1H-pyrazol-4-yl)-4-phenyl-1H-pyrrol-2-yl)acetamide Io. 


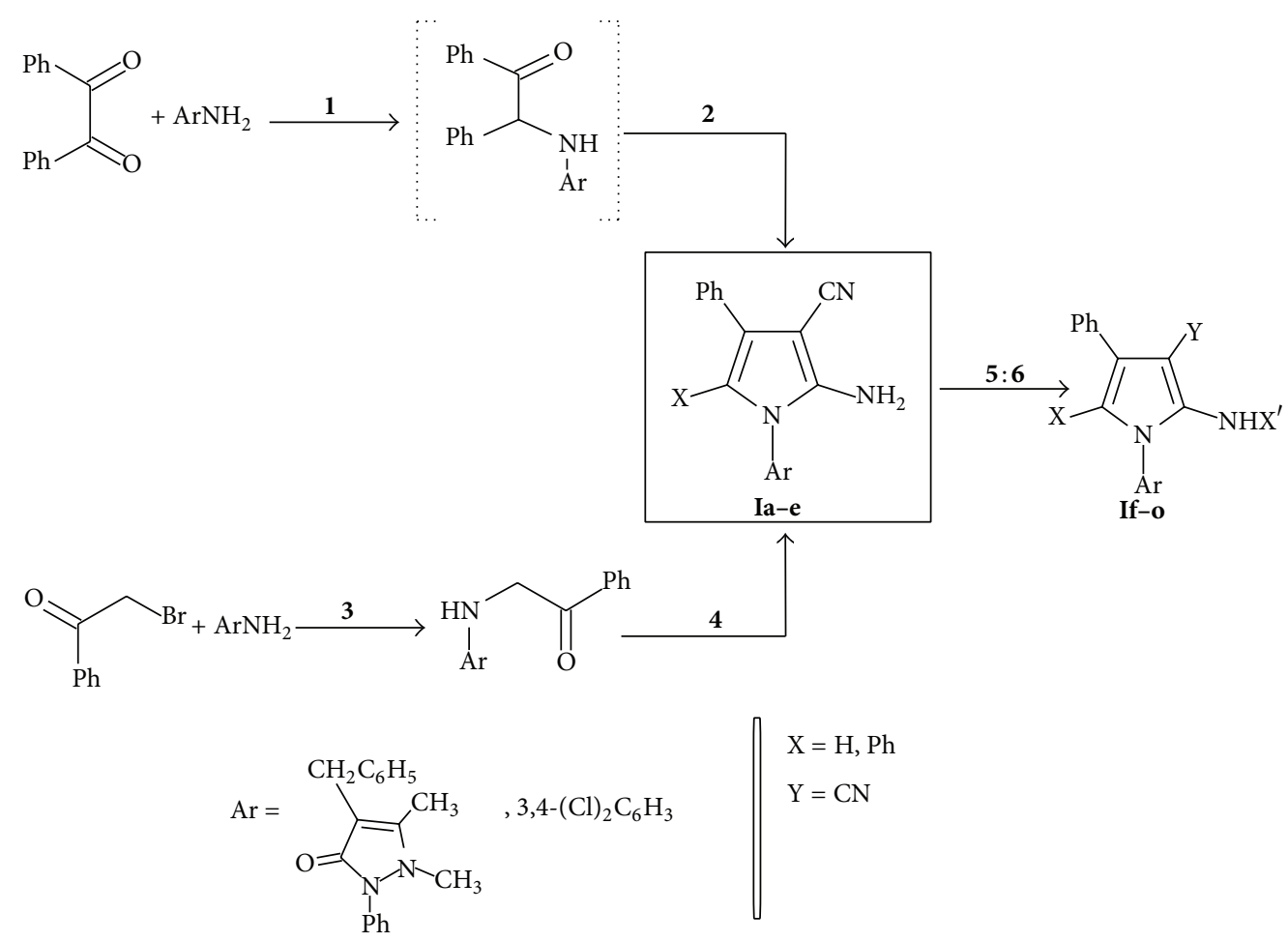

Scheme 1: Synthetic pathways for compounds Ia-o: reagents and conditions: (1) pyridine/benzene; (2) $\mathrm{CH}_{2}(\mathrm{CN})_{2} ;(3) \mathrm{NaHCO}_{3} / \mathrm{EtOH}(\mathbf{4})$ $\mathrm{CH}_{2}(\mathrm{CN})_{2} / \mathrm{NaOEt}$; (5) TEOF; or (6) $\mathrm{Ac}_{2} \mathrm{O}$.

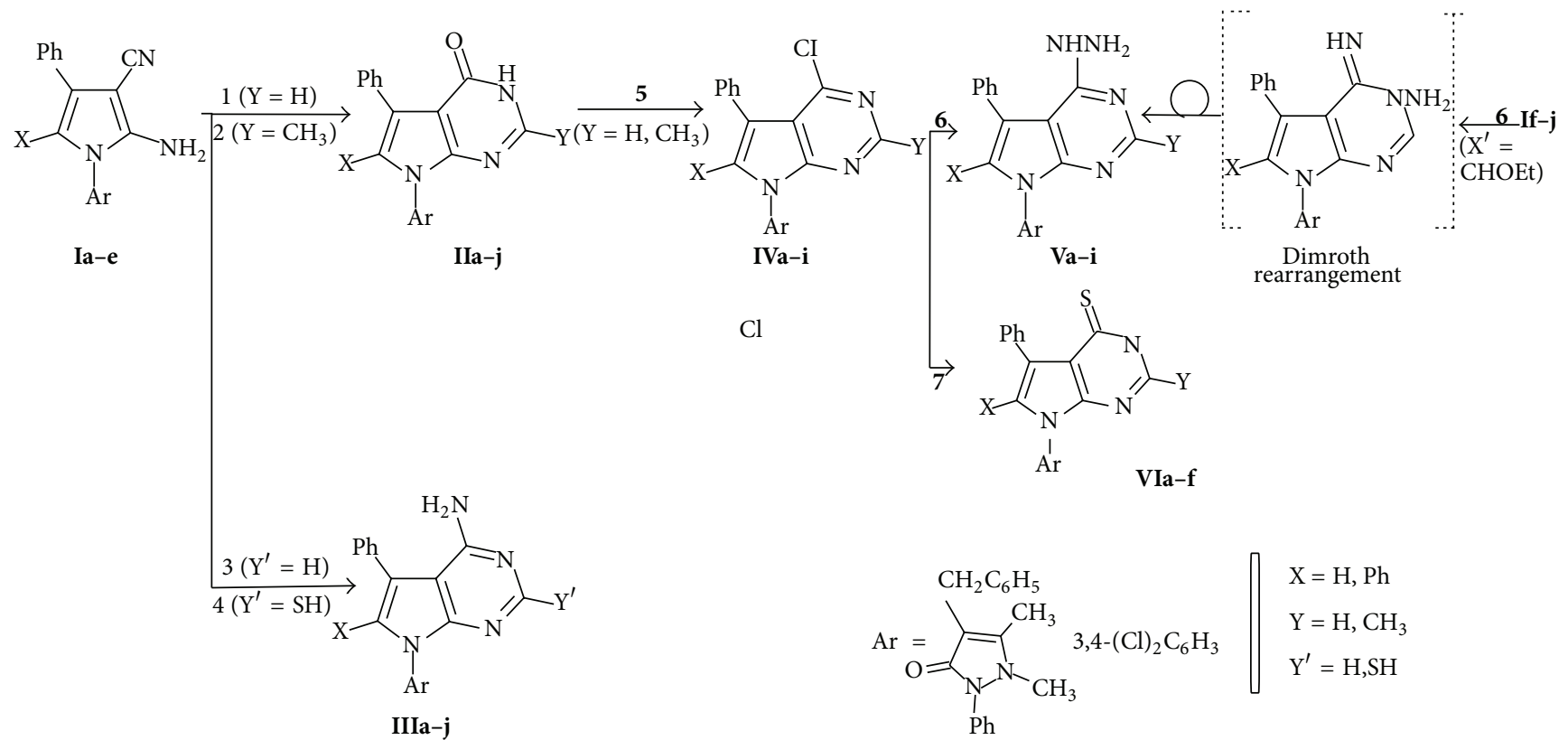

Scheme 2: Synthetic pathways for compounds I-VI: reagents and conditions: (1) $\mathrm{HCO}_{2} \mathrm{H}$; (2) $\mathrm{AcOH} / \mathrm{HCl}$; (3) $\mathrm{HCONH}_{2}$; (4) $\mathrm{NH}_{2} \mathrm{CSNH}_{2}$; (5) $\mathrm{POCl}_{3} ;(6) \mathrm{N}_{2} \mathrm{H}_{4} \cdot \mathrm{H}_{2} \mathrm{O}$; and (7) $\mathrm{NH}_{2} \mathrm{CSNH}_{2}$.

Yield: $72 \%$; M.P. $135-138^{\circ} \mathrm{C} ;{ }^{1} \mathrm{H}$ NMR (DMSO- $\left.d_{6}, 300 \mathrm{MHz}\right)$ $\delta(\mathrm{ppm}): 2.23\left(\mathrm{~s}, 3 \mathrm{H}, \mathrm{CO}-\mathrm{CH}_{3}\right), 2.43\left(\mathrm{~s}, 3 \mathrm{H}, \mathrm{CH}_{3}\right), 3.12(\mathrm{~s}$, $\left.3 \mathrm{H}, \mathrm{N}-\mathrm{CH}_{3}\right), 7.0-7.8\left(\mathrm{~m}, 11 \mathrm{H}, \mathrm{Ar}-\mathrm{H}\right.$ and $\left.\mathrm{C}_{6}-\mathrm{H}\right), 9.4(\mathrm{~s}, 1 \mathrm{H}$, $\mathrm{NH}, \mathrm{D}_{2} \mathrm{O}$ exchangeable); IR $(\mathrm{KBr}) v\left(\mathrm{~cm}^{-1}\right): 3350(\mathrm{NH})$, $2310(\mathrm{C} \equiv \mathrm{N}), 1715,1705(\mathrm{C}=\mathrm{O})$; MS (EI) $m / z: 411\left(\mathrm{M}^{+}, 15.4 \%\right)$, $412\left(\mathrm{M}^{+}+1,3.73 \%\right)$; Anal. Calcd for $\mathrm{C}_{24} \mathrm{H}_{21} \mathrm{~N}_{5} \mathrm{O}_{2}$ (411.17): C,
70.06; H, 5.14; N, 17.02; O, 7.78\%. Found: C, 70.37; H, 5.45; N, $17.34 ; \mathrm{O}, 7.95 \%$.

2.1.9. 7-(2,4-Dimethyl-5-oxo-1-phenyl-2,5-dihydro-1H-pyrazol-3-yl)-5,6-diphenyl -3H-pyrrolo[2,3-d]pyrimidin-4(7H)one IId,e (Scheme 2). Compound Id or e (0.01 mol) in formic 


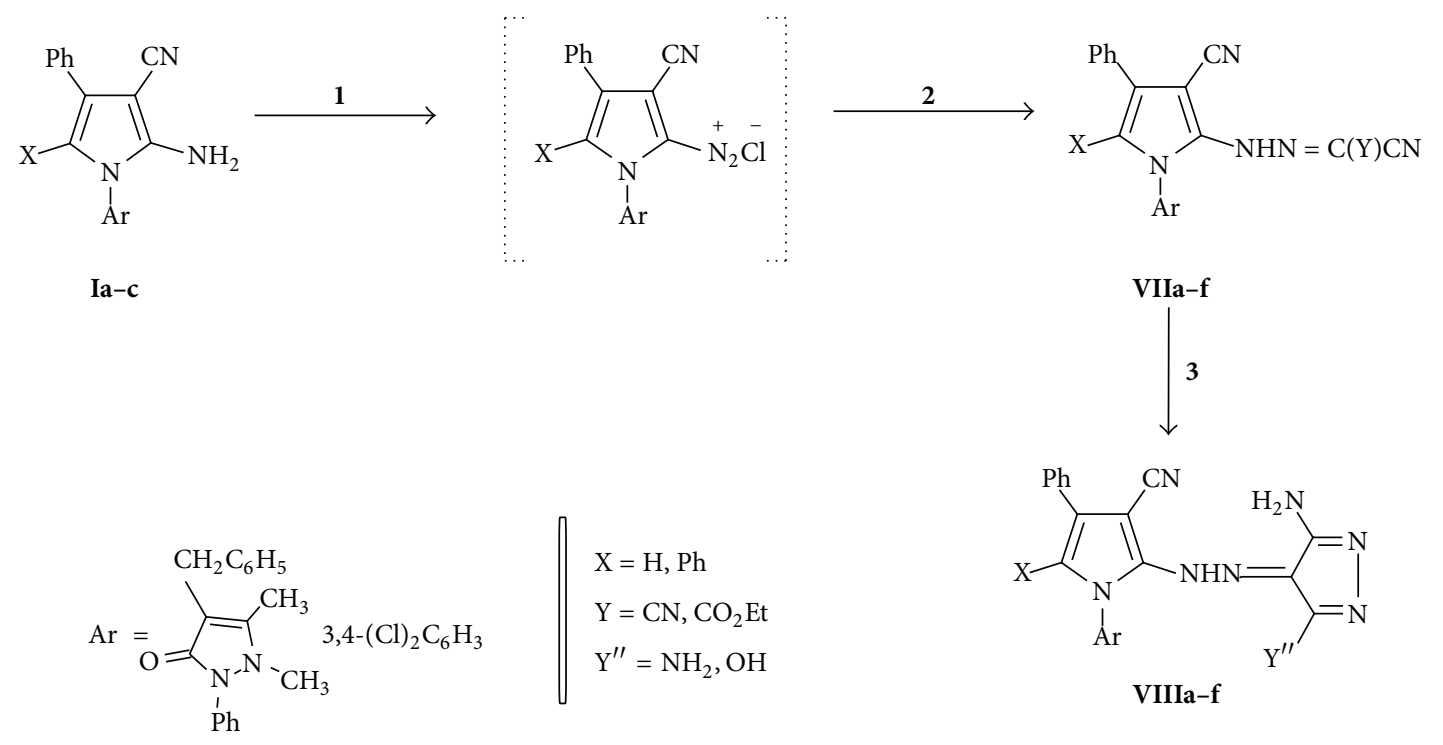

Scheme 3: Synthetic pathways for compounds VII-VIII: reagents and conditions: (1) $\left.\mathrm{NaNO}_{2} / \mathrm{HCl}_{/} / \mathrm{Stirring}^{(75} \mathrm{min}\right)$; $\mathrm{NCCH}_{2} \mathrm{Y} / \mathrm{CH}_{3} \mathrm{CO}_{2} \mathrm{NH}_{4} / \mathrm{EtOH}$; and (3) $\mathrm{N}_{2} \mathrm{H}_{4} \cdot \mathrm{H}_{2} \mathrm{O}$.

acid $(20 \mathrm{~mL}, 85 \%)$ was refluxed for $12 \mathrm{~h}$. The reaction mixture was cooled, poured onto ice/water to give a precipitate which was filtered, dried, and recrystallized from ethanol to give the target compounds IId, e.

7-(3,4-Dichlorophenyl)-5,6-diphenyl-3H-pyrrolo[2,3-d]pyrimidin-4(7H)-one IId. Yield: 56\%; M.P. $172-176^{\circ} \mathrm{C} ;{ }^{1} \mathrm{H}$ NMR $\left(\mathrm{DMSO}-d_{6}, 300 \mathrm{MHz}\right) \delta$ (ppm): 6.9-7.8 (m, 13H, Ar-H), $8.4\left(\mathrm{~s}, 1 \mathrm{H}, \mathrm{C}_{2}-\mathrm{H}\right), 12.40\left(\mathrm{~s}, 1 \mathrm{H}, \mathrm{NH}, \mathrm{D}_{2} \mathrm{O}\right.$ exchangeable): ${ }^{13} \mathrm{C}$ NMR (DMSO- $d_{6}$ ): $\delta 118.24,119.37,125.8,126.18,127.80$, $128.45,129.84,130.29,132.16,132.70,133.62,134.96,138.25$, 142.05, 146.2, 163.15 ppm; IR $(\mathrm{KBr}) v\left(\mathrm{~cm}^{-1}\right): 3410,3350$ $\left(\mathrm{NH}_{2}\right), 1720,1705(\mathrm{C}=\mathrm{O}), 1550(\mathrm{C}=\mathrm{N})$; $\mathrm{MS}(\mathrm{EI}) \mathrm{m} / z: 432\left(\mathrm{M}^{+}\right.$, 21.5\%), $434\left(\mathrm{M}^{+}+2,13.9 \%\right), 436\left(\mathrm{M}^{+}+4,4.2 \%\right)$; Anal. Calcd for $\mathrm{C}_{24} \mathrm{H}_{15} \mathrm{Cl}_{2} \mathrm{~N}_{3} \mathrm{O}$ (432.30): C, 66.68; $\mathrm{H}, 3.50 ; \mathrm{Cl}, 16.40 ; \mathrm{N}$, 9.72; O, 3.70\%. Found: C, 66.32; H, 3.18; Cl, 16.21; N, 9.50; O, $3.46 \%$.

7-(1,5-Dimethyl-3-oxo-2-phenyl-2,3-dihydro-1H-pyrazol-4-yl) -5-phenyl-3H-pyrrolo[2,3-d]pyrimidin-4(7H)-one IIe. Yield: 65\%; M.P. $205-208^{\circ} \mathrm{C}$; ${ }^{1} \mathrm{H}$ NMR (DMSO- $\left.d_{6}, 300 \mathrm{MHz}\right) \delta$ (ppm): 2.41 (s, 3H, $\left.\mathrm{CH}_{3}\right), 3.21\left(\mathrm{~s}, 3 \mathrm{H}, \mathrm{N}-\mathrm{CH}_{3}\right), 6.9-7.8(\mathrm{~m}$, $11 \mathrm{H}, \mathrm{Ar}-\mathrm{H}$ and $\left.\mathrm{C}_{6}-\mathrm{H}\right), 8.32\left(\mathrm{~s}, 1 \mathrm{H}, \mathrm{C}_{2}-\mathrm{H}\right), 12.32$ (s, $1 \mathrm{H}, \mathrm{NH}$, $\mathrm{D}_{2} \mathrm{O}$ exchangeable); IR $(\mathrm{KBr}) v\left(\mathrm{~cm}^{-1}\right): 3410,3320\left(\mathrm{NH}_{2}\right)$, $1720,1680(\mathrm{C}=\mathrm{O}), 1560(\mathrm{C}=\mathrm{N})$ and disappearance of the $\mathrm{CN}$ group; MS (EI) $m / z$ : $379\left(\mathrm{M}^{+}, 23.5 \%\right), 380\left(\mathrm{M}^{+}+1,7.1 \%\right)$; Anal. Calcd for $\mathrm{C}_{23} \mathrm{H}_{19} \mathrm{~N}_{5} \mathrm{O}_{2}$ (379.43): C, 69.51; $\mathrm{H}, 4.82 ; \mathrm{N}$, 17.62; O, 8.05\%. Found: C, 69.89; H, 5.16; N, 17.97; O, 8.41\%.

2.1.10. 7-Disubstituted-2-methyl-5-phenyl-7H-pyrrolo[2,3-d] pyrimidin-4(3H)-one IIi, $\boldsymbol{j}$ (Scheme 2). Compound Id or e $(0.01 \mathrm{~mol})$ in acetic acid/HCl $(3: 1)(30 \mathrm{~mL})$ was refluxed for $12 \mathrm{~h}$. The reaction mixture was cooled, poured onto ice/water, neutralized with ammonia to give a precipitate which was filtered, dried, and recrystallized from methanol to give the target compounds II i, j.

7-(3,4-Dichlorophenyl)-2-methyl-5,6-diphenyl-3H-pyrrolo[2, 3-d]pyrimidin-4(7H)-one II i. Yield: 67\%; M.P. $200-202^{\circ} \mathrm{C}$; ${ }^{1} \mathrm{H}$ NMR (DMSO- $\left.d_{6}, 300 \mathrm{MHz}\right) \delta(\mathrm{ppm}): 2.35(\mathrm{~s}, 3 \mathrm{H}$, $\left.\mathrm{C}_{2}-\mathrm{CH}_{3}\right), 6.9-7.8(\mathrm{~m}, 13 \mathrm{H}, \mathrm{Ar}-\mathrm{H}), 12.40\left(\mathrm{~s}, 1 \mathrm{H}, \mathrm{NH}, \mathrm{D}_{2} \mathrm{O}\right.$ exchangeable); IR (KBr) $v\left(\mathrm{~cm}^{-1}\right): 3430,3330\left(\mathrm{NH}_{2}\right), 1710$ $(\mathrm{C}=\mathrm{O}), 1570(\mathrm{C}=\mathrm{N})$; MS (EI) $m / z: 445\left(\mathrm{M}^{+}, 26.2 \%\right), 447$ $\left(\mathrm{M}^{+}+2,15.3 \%\right), 449\left(\mathrm{M}^{+}+4,1.04 \%\right)$; Anal. Calcd for $\mathrm{C}_{25} \mathrm{H}_{17} \mathrm{Cl}_{2} \mathrm{~N}_{3} \mathrm{O}$ (445.07): C, 67.28; H, 3.84; Cl, 15.89; N, 9.41; $\mathrm{O}, 3.58 \%$. Found: C, 67.45; H, 4.11; Cl, 16.22; N, 9.74; O, 3.82\%.

7-(1,5-Dimethyl-3-oxo-2-phenyl-2,3-dihydro-1H-pyrazol-4-yl) -2-methyl-5-phenyl-3H-pyrrolo[2,3-d]pyrimidin-4(7H)-one II j. Yield: 67\%; M.P. $225-227^{\circ} \mathrm{C}$; ${ }^{1} \mathrm{H}$ NMR (DMSO- $d_{6}$, $300 \mathrm{MHz}) \delta(\mathrm{ppm}): 2.32\left(\mathrm{~s}, 3 \mathrm{H}, \mathrm{C}_{2}-\mathrm{CH}_{3}\right), 2.41\left(\mathrm{~s}, 3 \mathrm{H}, \mathrm{CH}_{3}\right)$, $3.22\left(\mathrm{~s}, 3 \mathrm{H}, \mathrm{N}-\mathrm{CH}_{3}\right), 7-7.8\left(\mathrm{~m}, 11 \mathrm{H}, \mathrm{Ar}-\mathrm{H}\right.$ and $\left.\mathrm{C}_{6}-\mathrm{H}\right), 12.36(\mathrm{~s}$, $1 \mathrm{H}, \mathrm{NH}, \mathrm{D}_{2} \mathrm{O}$ exchangeable); IR $(\mathrm{KBr}) v\left(\mathrm{~cm}^{-1}\right): 3430,3330$ $\left(\mathrm{NH}_{2}\right), 1730,1700(\mathrm{C}=\mathrm{O}), 1560(\mathrm{C}=\mathrm{N}) ; \mathrm{MS}(\mathrm{EI}) \mathrm{m} / z: 487$ $\left(\mathrm{M}^{+}, 45 \%\right), 488\left(\mathrm{M}^{+}+1,15.2 \%\right)$; Anal. Calcd for $\mathrm{C}_{24} \mathrm{H}_{21} \mathrm{~N}_{5} \mathrm{O}_{2}$ (411.46): C, 70.06; H, 5.14; N, 17.02; O, 7.78\%. Found: C, 70.24; $\mathrm{H}, 5.32 ; \mathrm{N}, 17.31 ; \mathrm{O}, 7.96 \%$.

2.1.11. 6,7-Disubstituted-5-phenyl-7H-pyrrolo[2,3-d]pyrimidin-4-ylamines IIId, $\boldsymbol{e}$ (Scheme 2). A suspension of the appropriate aminopyrrole $\mathbf{I}, \mathbf{d}$, or $\mathbf{e}(0.01 \mathrm{~mol})$ and formamide $(30 \mathrm{~mL}, 0.066 \mathrm{~mol})$ were heated under reflux for $9 \mathrm{~h}$, cooled, poured onto ice/water to give precipitates which were filtered off, dried, and recrystallized from ethanol to give the target compounds III d, e.

7-(3,4-Dichlorophenyl)-5,6-diphenyl-7H-pyrrolo[2,3-d]pyrim-idin-4-amine III $\boldsymbol{d}$. Yield: 63\%; M.P. $115-118^{\circ} \mathrm{C} ;{ }^{1} \mathrm{H}$ NMR $\left(\mathrm{DMSO}-d_{6}, 300 \mathrm{MHz}\right) \delta$ (ppm): 5.4 (brs, $2 \mathrm{H}, \mathrm{NH}_{2}, \mathrm{D}_{2} \mathrm{O}$ 
exchangeable), 7.1-7.7 (m, 13H, Ar-H), $8.2\left(\mathrm{~s}, 1 \mathrm{H}, \mathrm{C}_{2}-\mathrm{H}\right)$; IR $(\mathrm{KBr}) v\left(\mathrm{~cm}^{-1}\right)$ : 3440, $3350\left(\mathrm{NH}_{2}\right), 1570(\mathrm{C}=\mathrm{N}), 1610(\mathrm{C}=\mathrm{C})$; MS (EI) $m / z: 430\left(\mathrm{M}^{+}, 31.2 \%\right), 432\left(\mathrm{M}^{+}+2,17.6 \%\right), 434\left(\mathrm{M}^{+}+\right.$ 4, 0.98\%); Anal. Calcd for $\mathrm{C}_{24} \mathrm{H}_{16} \mathrm{Cl}_{2} \mathrm{~N}_{4}$ (430.08): C, 66.83; $\mathrm{H}, 3.74 ; \mathrm{Cl}, 16.44 ; \mathrm{N}, 12.99 \%$. Found: C, 67.07; H, 5.11; Cl, $16.81 ; \mathrm{N}, 13.36 \%$.

4-(4-Amino-5-phenyl-7H-pyrrolo[2,3-d]pyrimidin-7-yl)-1,5dimethyl-2-phenyl-1H-pyrazol-3(2H)-one III e. Yield: 69\%; M.P. $152-155^{\circ} \mathrm{C} ;{ }^{1} \mathrm{H}$ NMR (DMSO- $\left.d_{6}, 300 \mathrm{MHz}\right) \delta$ (ppm): $2.41\left(\mathrm{~s}, 3 \mathrm{H}, \mathrm{CH}_{3}\right), 3.14$ (s, $\left.3 \mathrm{H}, \mathrm{N}-\mathrm{CH}_{3}\right), 5.8$ (brs, $2 \mathrm{H}, \mathrm{NH}_{2}$, $\mathrm{D}_{2} \mathrm{O}$ exchangeable), 7.1-7.8 (m, $11 \mathrm{H}, \mathrm{Ar}-\mathrm{H}$, and $\left.\mathrm{C}_{6}-\mathrm{H}\right), 8.3$ $\left(\mathrm{s}, 1 \mathrm{H}, \mathrm{C}_{2}-\mathrm{H}\right)$; IR $(\mathrm{KBr}) v\left(\mathrm{~cm}^{-1}\right): 3430,3330\left(\mathrm{NH}, \mathrm{NH}_{2}\right)$, $1705(\mathrm{C}=\mathrm{O}), 1600(\mathrm{C}=\mathrm{C}), 1540(\mathrm{C}=\mathrm{N})$; MS (EI) $m / z: 396$ $\left(\mathrm{M}^{+}, 13.7 \%\right), 397\left(\mathrm{M}^{+}+1,1.91 \%\right)$; Anal. Calcd for $\mathrm{C}_{23} \mathrm{H}_{20} \mathrm{~N}_{6} \mathrm{O}$ (396.44): C, 69.68; H, 5.08; N, 21.20; O, 4.04\%. Found: C, 69.42; H, 4.92; N, 20.89; O, 4.32\%.

2.1.12. 5-(5,6-Diphenyl-4-thioxo-3H-pyrrolo[2,3-d]pyrimidin7(4H)-yl)-1,4-dimethyl-2-phenyl-1H-pyrazol-3(2H)-one IIIi, $\boldsymbol{j}$ (Scheme 2). Compound I, d, or e $(0.01 \mathrm{~mol})$ and thiourea $(1.2 \mathrm{~g}, 0.02 \mathrm{~mol})$ were refluxed in dry ethanol $(20 \mathrm{~mL})$ for $12 \mathrm{~h}$. The reaction mixture was evaporated under reduced pressure and the residues were recrystallized from methanol to give the target compounds III $\mathbf{i}, \mathbf{j}$.

4-Amino-7-(3,4-dichlorophenyl)-5,6-diphenyl-1H-pyrrolo[2,3d] pyrimidine-2(7H)-thione IIIi. Yield: $70 \%$; M.P. $90-95^{\circ} \mathrm{C}$; ${ }^{1} \mathrm{H}$ NMR (DMSO- $\left.d_{6}, 300 \mathrm{MHz}\right) \delta(\mathrm{ppm}): 6.62\left(\mathrm{~s}, 2 \mathrm{H}, \mathrm{NH}_{2}\right.$, $\mathrm{D}_{2} \mathrm{O}$ exchangeable), 7.1-7.9 (m, 13H, Ar- $\left.\mathrm{H}\right), 9.22(\mathrm{~s}, 1 \mathrm{H}, \mathrm{NH}$, $\mathrm{D}_{2} \mathrm{O}$ exchangeable); IR $(\mathrm{KBr}) v\left(\mathrm{~cm}^{-1}\right): 3440,3340(\mathrm{NH}$, $\left.\mathrm{NH}_{2}\right), 1610(\mathrm{NH}-\mathrm{C}=\mathrm{S})$; MS (EI) $\mathrm{m} / z: 463\left(\mathrm{M}^{+}, 22.36 \%\right)$, $465\left(\mathrm{M}^{+}+2,14.23 \%\right), 467\left(\mathrm{M}^{+}+4,4.54 \%\right)$; Anal. Calcd for $\mathrm{C}_{24} \mathrm{H}_{16} \mathrm{Cl}_{2} \mathrm{~N}_{4} \mathrm{~S}$ (463.38): C, 62.21; H, 3.48; Cl, 15.30; N, 12.09; S, 6.92\%. Found: C, 62.45; H, 3.62; Cl, 15.49; N, 12.41; S, 7.21\%.

4-(4-Amino-5-phenyl-2-thioxo-1H-pyrrolo[2,3-d]pyrimidin7(2H)-yl)-1,5-dimethyl-2-phenyl-1H-pyrazol-3(2H)-one IIIj. Yield: $74 \%$; M.P. $172-174^{\circ} \mathrm{C} ;{ }^{1} \mathrm{H}$ NMR (DMSO- $d_{6}, 300 \mathrm{MHz}$ ) $\delta$ (ppm): 2.34 (s, $\left.3 \mathrm{H}, \mathrm{CH}_{3}\right), 3.11$ (s, $\left.3 \mathrm{H}, \mathrm{N}-\mathrm{CH}_{3}\right), 6.51$ (brs, $2 \mathrm{H}$, $\mathrm{NH}_{2}, \mathrm{D}_{2} \mathrm{O}$ exchangeable), 7.1-7.8 $\left(\mathrm{m}, 11 \mathrm{H}, \mathrm{Ar}-\mathrm{H}\right.$, and $\left.\mathrm{C}_{6}-\mathrm{H}\right)$, $8.3\left(\mathrm{~s}, 1 \mathrm{H}, \mathrm{C}_{2}-\mathrm{H}\right), 8.92\left(\mathrm{~s}, 1 \mathrm{H}, \mathrm{NH}, \mathrm{D}_{2} \mathrm{O}\right.$ exchangeable); IR $(\mathrm{KBr}) v\left(\mathrm{~cm}^{-1}\right): 3440,3370\left(\mathrm{NH}, \mathrm{NH}_{2}\right), 1610(\mathrm{C}=\mathrm{C}), 1600$ $(\mathrm{NH}-\mathrm{C}=\mathrm{S}) ; \mathrm{MS}(\mathrm{EI}) \mathrm{m} / z: 428\left(\mathrm{M}^{+}, 14.8 \%\right), 429\left(\mathrm{M}^{+}+1\right.$, 2.31\%); Anal. Calcd for $\mathrm{C}_{23} \mathrm{H}_{20} \mathrm{~N}_{6} \mathrm{OS}$ (428.51): C, 64.47; $\mathrm{H}$, 4.70 ; N, 19.61; O, 3.73; S, 7.48\%. Found: C, 64.78; H, 4.97; N, $19.92 ;$ O, 3.91; S, 7.62\%.

2.1.13. General Procedure for the Preparation of 4Chloropyrrolopyrimidines IV $\boldsymbol{d}-\mathbf{j}$ (Scheme 2). The appropriate compound II $(0.01 \mathrm{~mol})$ was refluxed in phosphorus oxychloride $(30 \mathrm{~mL})$ for $12 \mathrm{~h}$. The solution was cooled and poured with stirring onto ice/water and the formed precipitated was filtered, washed several times with water, dried, and recrystallized from ethanol to give the target compounds IVd-j.
4-Chloro-7-(3,4-dichlorophenyl)-5,6-diphenyl-7H-pyrrolo[2, 3-d]pyrimidine IVd. Yield: $76 \%$; M.P. $124-128^{\circ} \mathrm{C} ;{ }^{1} \mathrm{H}$ NMR (DMSO- $\left.d_{6}, 300 \mathrm{MHz}\right) \delta(\mathrm{ppm}): 6.9-7.8(\mathrm{~m}, 13 \mathrm{H}, \mathrm{Ar}-\mathrm{H}), 8.6$ $\left(\mathrm{s}, 1 \mathrm{H}, \mathrm{C}_{2}-\mathrm{H}\right) ;{ }^{13} \mathrm{C}$ NMR (DMSO- $\left.d_{6}\right): \delta 117.5,118.8,126.18$, $127.80,128.45,129.84,130.21,132.46,132.64,133.76,134.84$, 138.27, 141.05, 150.2, 151.24, $153.8 \mathrm{ppm}$; IR $(\mathrm{KBr}) v\left(\mathrm{~cm}^{-1}\right)$ : 3080, 2840(CH), $1612(\mathrm{C}=\mathrm{C}), 1580(\mathrm{C}=\mathrm{N})$; MS (EI) $m / z: 449$ $\left(\mathrm{M}^{+}, 29.98 \%\right), 451\left(\mathrm{M}^{+}+2,23.6 \%\right), 453\left(\mathrm{M}^{+}+4,6.9 \%\right), 455$ $\left(\mathrm{M}^{+}+6,0.66 \%\right)$; Anal. Calcd for $\mathrm{C}_{24} \mathrm{H}_{14} \mathrm{Cl}_{3} \mathrm{~N}_{3}$ (449.03): $\mathrm{C}$, $63.95 ; \mathrm{H}, 3.13 ; \mathrm{Cl}, 23.60 ; \mathrm{N}, 9.32 \%$. Found: C, 64.23; H, 3.42; $\mathrm{Cl}, 23.91 ; \mathrm{N}, 9.49 \%$.

4-(4-Chloro-5-phenyl-7H-pyrrolo[2,3-d]pyrimidin-7-yl)-1,5dimethyl-2-phenyl-1H-pyrazol-3(2H)-one IVe. Yield: 76\%; M.P. $125-130^{\circ} \mathrm{C} ;{ }^{1} \mathrm{H}$ NMR (DMSO- $\left.d_{6}, 300 \mathrm{MHz}\right) \delta(\mathrm{ppm})$ : $2.43\left(\mathrm{~s}, 3 \mathrm{H}, \mathrm{CH}_{3}\right), 3.12\left(\mathrm{~s}, 3 \mathrm{H}, \mathrm{N}-\mathrm{CH}_{3}\right), 6.9-7.8(\mathrm{~m}, 11 \mathrm{H}, \mathrm{Ar}-\mathrm{H}$ and $\left.\mathrm{C}_{6}-\mathrm{H}\right), 8.5\left(\mathrm{~s}, 1 \mathrm{H}, \mathrm{C}_{2}-\mathrm{H}\right)$; IR $(\mathrm{KBr}) v\left(\mathrm{~cm}^{-1}\right): 3080,2840$ (CH), $1730(\mathrm{C}=\mathrm{O}), 1612(\mathrm{C}=\mathrm{C}), 1580(\mathrm{C}=\mathrm{N}) ; \mathrm{MS}(\mathrm{EI}) \mathrm{m} / z: 415$ $\left(\mathrm{M}^{+}, 20 \%\right), 417\left(\mathrm{M}^{+}+2,5.5 \%\right)$; Anal. Calcd for $\mathrm{C}_{23} \mathrm{H}_{18} \mathrm{ClN}_{5} \mathrm{O}$ (415.87): C, 66.43; H, 4.36; Cl, 8.52; N, 16.84; O, 3.85\%. Found: C, 66.67; H, 4.71; Cl, 8.68; N, 16.94; O, 3.99\%.

7-Benzyl-4-chloro-2-methyl-5,6-diphenyl-7H-pyrrolo[2,3-d] pyrimidine IVf. Yield: $46 \%$; M.P. $120-124^{\circ} \mathrm{C} ;{ }^{1} \mathrm{H}$ NMR $\left(\mathrm{DMSO}-d_{6}, 300 \mathrm{MHz}\right) \delta(\mathrm{ppm}): 2.32\left(\mathrm{~s}, 3 \mathrm{H}, \mathrm{CH}_{3}\right), 5.12(\mathrm{~s}$, $\left.2 \mathrm{H}, \mathrm{Ph}-\mathrm{CH}_{2}\right), 6.9-7.8\left(\mathrm{~m}, 15 \mathrm{H}, \mathrm{Ar}-\mathrm{H}\right.$ and $\left.\mathrm{C}_{6}-\mathrm{H}\right)$; IR $(\mathrm{KBr})$ $v\left(\mathrm{~cm}^{-1}\right): 3080,2840(\mathrm{CH}), 1610(\mathrm{C}=\mathrm{C}), 1560(\mathrm{C}=\mathrm{N})$; MS (EI) $m / z: 409\left(\mathrm{M}^{+}, 14 \%\right), 411\left(\mathrm{M}^{+}+2,4.5 \%\right)$; Anal. Calcd for $\mathrm{C}_{26} \mathrm{H}_{20} \mathrm{ClN}_{3}$ (409.91): C, 76.18; H, 4.92; Cl, 8.65; N, 10.25\%. Found: C, 76.47; H, 5.23; Cl, 8.96; N, 10.59\%.

4-(4-Chloro-2-methyl-5,6-diphenyl-7H-pyrrolo[2,3-d]pyrimidin-7-yl)-1,5-dimethyl-2-phenyl-1H-pyrazol-3(2H)-one IVg. Yield: $42 \%$; M.P. $158-160^{\circ} \mathrm{C} ;{ }^{1} \mathrm{H}$ NMR (DMSO- $d_{6}, 300 \mathrm{MHz}$ ) $\delta$ (ppm): $2.30-2.44\left(\mathrm{~s}, 6 \mathrm{H}, 2^{*} \mathrm{CH}_{3}\right), 3.13\left(\mathrm{~s}, 3 \mathrm{H}, \mathrm{N}-\mathrm{CH}_{3}\right)$, 6.9-7.8 (m, 15H, Ar-H); IR (KBr) $v\left(\mathrm{~cm}^{-1}\right): 3080,2840(\mathrm{CH})$, $1710(\mathrm{C}=\mathrm{O}), 1605(\mathrm{C}=\mathrm{C}), 1577(\mathrm{C}=\mathrm{N})$; MS (EI) $\mathrm{m} / z: 505\left(\mathrm{M}^{+}\right.$, 13\%), $507\left(\mathrm{M}^{+}+2,2.5 \%\right)$; Anal. Calcd for $\mathrm{C}_{30} \mathrm{H}_{24} \mathrm{ClN}_{5} \mathrm{O}$ (505.17): C, 71.21; H, 4.78; Cl, 7.01; N, 13.84; O, 3.16\%. Found: C, $71.02 ; \mathrm{H}, 4.97 ; \mathrm{Cl}, 7.23 ; \mathrm{N}, 14.06 ; \mathrm{O}, 3.45 \%$.

4-Chloro-7-(3,4-dichlorophenyl)-2-methyl-5,6-diphenyl-7Hpyrrolo[2,3-d]pyrimidine IVh. Yield: $45 \%$; M.P. $108-110^{\circ} \mathrm{C}$; ${ }^{1} \mathrm{H}$ NMR (DMSO- $\left.d_{6}, 300 \mathrm{MHz}\right) \delta(\mathrm{ppm}): 2.36\left(\mathrm{~s}, 3 \mathrm{H}, \mathrm{CH}_{3}\right)$, 7.2-7.8 (m, 13H, Ar-H); IR (KBr) $v\left(\mathrm{~cm}^{-1}\right): 3080,2840(\mathrm{CH})$, $1600(\mathrm{C}=\mathrm{C}), 1565(\mathrm{C}=\mathrm{N})$; MS (EI) $\mathrm{m} / z: 464\left(\mathrm{M}^{+}, 11 \%\right), 466$ $\left(\mathrm{M}^{+}+2,6.5 \%\right), 468\left(\mathrm{M}^{+}+4,2.3 \%\right), 394\left(\mathrm{M}^{+}+6,0.88 \%\right)$; Anal. Calcd for $\mathrm{C}_{25} \mathrm{H}_{16} \mathrm{Cl}_{3} \mathrm{~N}_{3}$ (464.77): C, 64.61; H, 3.47; $\mathrm{Cl}, 22.88 ; \mathrm{N}, 9.04 \%$. Found: C, 64.99; H, 3.73; $\mathrm{Cl}, 23.08 ; \mathrm{N}$, $9.29 \%$.

4-(4-Chloro-2-methyl-5-phenyl-7H-pyrrolo[2,3-d]pyrimidin7-yl)-1,5-dimethyl-2-phenyl-1H-pyrazol-3(2H)-one IVi. Yield: $42 \%$; M.P. $95-100^{\circ} \mathrm{C} ;{ }^{1} \mathrm{H}$ NMR (DMSO- $\left.d_{6}, 300 \mathrm{MHz}\right) \delta$ (ppm): $2.28-2.46\left(\mathrm{~s}, 6 \mathrm{H}, 2^{*} \mathrm{CH}_{3}\right), 3.27\left(\mathrm{~s}, 3 \mathrm{H}, \mathrm{N}-\mathrm{CH}_{3}\right)$, 7.0-7.8 (m, $11 \mathrm{H}, \mathrm{Ar}-\mathrm{H}$ and $\left.\mathrm{C}_{6}-\mathrm{H}\right) ; \mathrm{IR}(\mathrm{KBr}) v\left(\mathrm{~cm}^{-1}\right)$ : 3070 , $2850(\mathrm{CH}), 1700(\mathrm{C}=\mathrm{O}), 1600(\mathrm{C}=\mathrm{C}), 1570(\mathrm{C}=\mathrm{N})$; MS (EI) $m / z: 429\left(\mathrm{M}^{+}, 16 \%\right), 431\left(\mathrm{M}^{+}+2,4.7 \%\right)$; Anal. Calcd for 
$\mathrm{C}_{24} \mathrm{H}_{20} \mathrm{ClN}_{5} \mathrm{O}$ (429.90): C, 67.05; H, 4.69; Cl, 8.25; N, 16.29; $\mathrm{O}, 3.72 \%$. Found: C, 67.42; H, 5.08; Cl, 8.57; N, 16.65; O, $4.02 \%$.

\subsubsection{General Procedure for the Preparation of 4-Hydrazino- pyrrolopyrimidines $\boldsymbol{V d}-\boldsymbol{i}$ (Scheme 2)}

Method A. Compound IV (0.01 mol) and hydrazine hydrate $(8 \mathrm{~mL}, 0.015 \mathrm{~mol}, 98 \%)$ were refluxed in dry ethanol $(30 \mathrm{~mL})$ for $12 \mathrm{~h}$. The solvent was removed under reduced pressure and the residues were recrystallized from methanol to give the target compounds $\mathbf{V}$.

Method B. Compounds Ii, j $(0.01 \mathrm{~mol})$ in dry toluene $(20 \mathrm{~mL})$ and hydrazine hydrate $(5 \mathrm{~mL}, 0.015 \mathrm{~mol}, 98 \%)$ were added with stirring at room temperature for $14 \mathrm{~h}$. The solvent was removed under reduced pressure, and the residue was recrystallized from methanol to give Vd, e; Compounds Vd, e prepared by this method are identical in all respects (physical and spectral data) to that prepared from Method A.

7-(3,4-Dichlorophenyl)-4-hydrazinyl-5,6-diphenyl-7H-pyrrolo [2,3-d]pyrimidine Vd. Yield: (A; 68\%, B; 56\%); M.P. 122$126^{\circ} \mathrm{C}$; ${ }^{1} \mathrm{H}$ NMR (DMSO- $\left.d_{6}, 300 \mathrm{MHz}\right) \delta$ (ppm): 4.9-5.2 (brs, $2 \mathrm{H}, \mathrm{NH}_{2}, \mathrm{D}_{2} \mathrm{O}$ exchangeable), 7.1-7.8 (m, 14H, Ar-H and $\mathrm{NH}, \mathrm{D}_{2} \mathrm{O}$ exchangeable), $8.3\left(\mathrm{~s}, 1 \mathrm{H}, \mathrm{C}_{2}-\mathrm{H}\right) ;{ }^{13} \mathrm{C} \mathrm{NMR}$ $\left(\mathrm{DMSO}-d_{6}\right): \delta 117.45,118.69,126.21,127.47,128.4,129.74$, $130.2,132.39,132.64,133.76,134.84,138.27,141.05,153.2,153.8$, $168.71 \mathrm{ppm}$; IR $(\mathrm{KBr}) v\left(\mathrm{~cm}^{-1}\right): 3420,3350\left(\mathrm{NH}_{2}\right) 3210(\mathrm{NH})$, $1610(\mathrm{C}=\mathrm{C}), 1580(\mathrm{C}=\mathrm{N})$; MS (EI) $m / z: 446\left(\mathrm{M}^{+}, 23 \%\right)$, $448\left(\mathrm{M}^{+}+2,14 \%\right), 450\left(\mathrm{M}^{+}+4,3.4 \%\right)$; Anal. Calcd for $\mathrm{C}_{24} \mathrm{H}_{17} \mathrm{Cl}_{2} \mathrm{~N}_{5}$ (446.33): C, 64.58; H, 3.84; Cl, 15.89; N, 15.69\%. Found: C, 64.95; H, 4.20; Cl, 16.23; N, 16.04\%.

4-(4-Hydrazinyl-5-phenyl-7H-pyrrolo[2,3-d]pyrimidin-7-yl) 1,5-dimethyl-2-phenyl-1H-pyrazol-3(2H)-one Ve. Yield: (A) $71 \%$, (B) $60 \%$; M.P. $142-146^{\circ} \mathrm{C}$; ${ }^{1} \mathrm{H}$ NMR (DMSO- $d_{6}$, $300 \mathrm{MHz}) \delta$ (ppm): $2.42\left(\mathrm{~s}, 3 \mathrm{H}, \mathrm{CH}_{3}\right), 3.13$ (s, 3H, N-CH$)_{3}$, 4.8-5.1 (brs, $2 \mathrm{H}, \mathrm{NH}_{2}, \mathrm{D}_{2} \mathrm{O}$ exchangeable), 7.1-7.8 (m, 11H, Ar$\mathrm{H}, \mathrm{C}_{6}-\mathrm{H}$ and $\mathrm{NH}, \mathrm{D}_{2} \mathrm{O}$ exchangeable), 8.2 (s, $\left.1 \mathrm{H}, \mathrm{C}_{2}-\mathrm{H}\right)$; IR $(\mathrm{KBr}) v\left(\mathrm{~cm}^{-1}\right): 3440,3330\left(\mathrm{NH}_{2}\right) 3260(\mathrm{NH}), 1705(\mathrm{C}=\mathrm{O})$, $1600(\mathrm{C}=\mathrm{C}), 1580(\mathrm{C}=\mathrm{N})$; MS (EI) $\mathrm{m} / z: 411\left(\mathrm{M}^{+}, 28 \%\right), 412$ $\left(\mathrm{M}^{+}+1,6.5 \%\right)$; Anal. Calcd for $\mathrm{C}_{23} \mathrm{H}_{21} \mathrm{~N}_{7} \mathrm{O}$ (411.46): C, 67.14; H, 5.14; N, 23.83; O, 3.89\%. Found: C, 67.52; H, 5.48; N, 24.15; $\mathrm{O}, 4.52 \%$.

7-Benzyl-4-hydrazinyl-2-methyl-5,6-diphenyl-7H-pyrrolo[2,3 -d] pyrimidine $\boldsymbol{V f}$. Yield: 67\%; M.P. $147-152^{\circ} \mathrm{C} ;{ }^{1} \mathrm{H}$ NMR $\left(\mathrm{DMSO}-d_{6}, 300 \mathrm{MHz}\right) \delta$ (ppm): $2.29\left(\mathrm{~s}, 3 \mathrm{H}, \mathrm{CH}_{3}\right), 4.9-5.2$ (brs, $2 \mathrm{H}, \mathrm{NH}_{2}, \mathrm{D}_{2} \mathrm{O}$ exchangeable), 5.78 (s, 2H, Ph- $\mathrm{CH}_{2}$ ), 7.23-7.8 (m, 16H, Ar- $\mathrm{H}$ and $\mathrm{NH}, \mathrm{D}_{2} \mathrm{O}$ exchangeable); IR $(\mathrm{KBr}) v\left(\mathrm{~cm}^{-1}\right): 3430,3350\left(\mathrm{NH}_{2}\right) 3250(\mathrm{NH}), 1605(\mathrm{C}=\mathrm{C})$, $1570(\mathrm{C}=\mathrm{N})$; MS (EI) $m / z: 405\left(\mathrm{M}^{+}, 29 \%\right), 406\left(\mathrm{M}^{+}+1\right.$, 4.1\%); Anal. Calcd for $\mathrm{C}_{26} \mathrm{H}_{23} \mathrm{~N}_{5}$ (405.49): C, 77.01; H, 5.72; N, 17.27\%. Found: C, 77.38; H, 6.05; N, 17.62\%.

4-(4-Hydrazinyl-2-methyl-5,6-diphenyl-7H-pyrrolo[2,3-d] pyrimidin-7-yl)-1,5-dimethyl-2-phenyl-1H-pyrazol-3(2H)-one Vg. Yield: 68\%; M.P. $185-188^{\circ} \mathrm{C}$; ${ }^{1} \mathrm{H}$ NMR (DMSO- $d_{6}$,
$300 \mathrm{MHz}) \delta(\mathrm{ppm}): 2.22-2.46\left(\mathrm{~s}, 6 \mathrm{H}, 2^{*} \mathrm{CH}_{3}\right), 3.11(\mathrm{~s}, 3 \mathrm{H}$, $\mathrm{N}-\mathrm{CH}_{3}$ ), 4.34-4.8 (brs, $2 \mathrm{H}, \mathrm{NH}_{2}, \mathrm{D}_{2} \mathrm{O}$ exchangeable), 7.2-7.8 $\left(\mathrm{m}, 16 \mathrm{H}, \mathrm{Ar}-\mathrm{H}\right.$ and $\mathrm{NH}, \mathrm{D}_{2} \mathrm{O}$ exchangeable): IR $(\mathrm{KBr}) v$ $\left(\mathrm{cm}^{-1}\right): 3420,3350\left(\mathrm{NH}_{2}\right) 3240(\mathrm{NH}), 1700(\mathrm{C}=\mathrm{O}), 1605$ $(\mathrm{C}=\mathrm{C}), 1560(\mathrm{C}=\mathrm{N})$; MS (EI) m/z: $501\left(\mathrm{M}^{+}, 31 \%\right), 502\left(\mathrm{M}^{+}+\right.$ 1, 5.4\%); Anal. Calcd for $\mathrm{C}_{30} \mathrm{H}_{27} \mathrm{~N}_{7} \mathrm{O}$ (501.58): C, 71.84; $\mathrm{H}$, 5.43; N, 19.55; O, 3.19\%. Found: C, 72.20; H, 5.81; N, 19.92; O, $3.55 \%$.

7-(3,4-Dichlorophenyl)-4-hydrazinyl-2-methyl-5,6-diphenyl7H-pyrrolo[2,3-d]pyrimidine Vh. Yield: 59\%; M.P. 142-146 ${ }^{\circ} \mathrm{C} ;{ }^{1} \mathrm{H}$ NMR (DMSO- $\left.d_{6}, 300 \mathrm{MHz}\right) \delta(\mathrm{ppm}): 2.25$ (s, 3H, $\mathrm{CH}_{3}$ ), 4.5-4.9 (brs, 2H, $\mathrm{NH}_{2}, \mathrm{D}_{2} \mathrm{O}$ exchangeable), 7.3-7.8 (m, 14H, Ar-H, NH, $\mathrm{D}_{2} \mathrm{O}$ exchangeable); IR (KBr) $v\left(\mathrm{~cm}^{-1}\right)$ : 3440, $3360\left(\mathrm{NH}_{2}\right) 3250(\mathrm{NH}), 1610(\mathrm{C}=\mathrm{C}), 1560(\mathrm{C}=\mathrm{N})$; MS (EI) $m / z: 460\left(\mathrm{M}^{+}, 31 \%\right), 462\left(\mathrm{M}^{+}+2,18 \%\right), 464\left(\mathrm{M}^{+}+4\right.$, 4.9\%); Anal. Calcd for $\mathrm{C}_{25} \mathrm{H}_{19} \mathrm{Cl}_{2} \mathrm{~N}_{5}$ (460.36): C, 65.22; $\mathrm{H}$, 4.16; Cl, 15.40; N, 15.21\%. Found: C, 65.57; H, 4.52; Cl, 15.78; $\mathrm{N}, 15.56 \%$.

4-(4-Hydrazinyl-2-methyl-5-phenyl-7H-pyrrolo[2,3d]pyrimidin-7-yl)-1,5-dimethyl-2-phenyl-1H-pyrazol-3(2H)one Vi. Yield: 69\%; M.P. $148-150^{\circ} \mathrm{C} ;{ }^{1} \mathrm{H}$ NMR (DMSO- $d_{6}$, $300 \mathrm{MHz}) \delta(\mathrm{ppm}): 2.23-2.44\left(\mathrm{~s}, 6 \mathrm{H}, 2^{*} \mathrm{CH}_{3}\right), 3.13(\mathrm{~s}, 3 \mathrm{H}$, $\mathrm{N}-\mathrm{CH}_{3}$ ), 4.4-4.78 (brs, $2 \mathrm{H}, \mathrm{NH}_{2}, \mathrm{D}_{2} \mathrm{O}$ exchangeable), 7.2-7.8 (m, $12 \mathrm{H}, \mathrm{Ar}-\mathrm{H}, \mathrm{C}_{6}-\mathrm{H}$ and $\mathrm{NH}, \mathrm{D}_{2} \mathrm{O}$ exchangeable); IR (KBr) $v\left(\mathrm{~cm}^{-1}\right):$ 3430, $3360\left(\mathrm{NH}_{2}\right) 3250(\mathrm{NH}), 1705(\mathrm{C}=\mathrm{O}), 1600$ $(\mathrm{C}=\mathrm{C}), 1580(\mathrm{C}=\mathrm{N})$; MS (EI) $\mathrm{m} / z$ : $501\left(\mathrm{M}^{+}, 31 \%\right), 502\left(\mathrm{M}^{+}+\right.$ 1, 5.4\%); Anal. Calcd for $\mathrm{C}_{24} \mathrm{H}_{23} \mathrm{~N}_{7} \mathrm{O}$ (425.49): C, 67.75; $\mathrm{H}$, 5.45; N, 23.04; O, 3.76\%. Found: C, 68.12; H, 5.76; N, 23.37; $\mathrm{O}, 4.11 \%$.

2.1.15. General Procedure for the Preparation of 4-Thienopyrrolopyrimidine VI $\boldsymbol{d}-\boldsymbol{f}$ (Scheme 2). Compound III $(0.01 \mathrm{~mol})$ and thiourea $(1.2 \mathrm{~g}, 0.02 \mathrm{~mol})$ were refluxed in dry ethanol $(20 \mathrm{~mL})$ for $14 \mathrm{~h}$. The reaction mixture was evaporated under reduced pressure and the residues were recrystallized from methanol to give the target compounds VI.

7-(3,4-Dichlorophenyl)-5,6-diphenyl-3H-pyrrolo[2,3-d]pyrimidine-4(7H)-thione VId. Yield: 66\%; M.P. $142-166^{\circ} \mathrm{C} ;{ }^{1} \mathrm{H}$ NMR (DMSO- $\left.d_{6}, 300 \mathrm{MHz}\right) \delta(\mathrm{ppm}): 7.3-7.8$ (m, 13H, Ar$\mathrm{H}), 9.02$ (s, $\left.1 \mathrm{H}, \mathrm{C}_{2}-\mathrm{H}\right), 11.71\left(\mathrm{~s}, 1 \mathrm{H}, \mathrm{NH}, \mathrm{D}_{2} \mathrm{O}\right.$ exchangeable); IR $(\mathrm{KBr}) v\left(\mathrm{~cm}^{-1}\right): 3250(\mathrm{NH}), 1630(\mathrm{NH}-\mathrm{C}=\mathrm{S}), 1560(\mathrm{C}=\mathrm{N})$; MS (EI) $m / z: 447\left(\mathrm{M}^{+}, 28 \%\right), 449\left(\mathrm{M}^{+}+2,18 \%\right), 451\left(\mathrm{M}^{+}+4\right.$, 0.98\%); Anal. Calcd for $\mathrm{C}_{24} \mathrm{H}_{15} \mathrm{Cl}_{2} \mathrm{~N}_{3} \mathrm{~S}$ (447.04): C, 64.29; $\mathrm{H}$, 3.37; Cl, 15.81; N, 9.37; S, 7.15\%. Found: C, 64.64; H, 3.74; Cl, $16.14 ; \mathrm{N}, 9.73 ; \mathrm{S}, 7.52 \%$.

1,5-Dimethyl-2-phenyl-4-(5-phenyl-4-thioxo-3H-pyrrolo[2,3d]pyrimidin-7(4H)-yl)-1H-pyrazol-3(2H)-one VIe. Yield: 61\%; M.P. $142-166^{\circ} \mathrm{C}$; ${ }^{1} \mathrm{H}$ NMR (DMSO-d, $\left.300 \mathrm{MHz}\right) \delta$ (ppm): $2.44\left(\mathrm{~s}, 3 \mathrm{H}, \mathrm{CH}_{3}\right), 3.13\left(\mathrm{~s}, 3 \mathrm{H}, \mathrm{N}-\mathrm{CH}_{3}\right), 7.2-7.8(\mathrm{~m}$, $11 \mathrm{H}, \mathrm{Ar}-\mathrm{H}$, and $\left.\mathrm{C}_{6}-\mathrm{H}\right) 9.12\left(\mathrm{~s}, 1 \mathrm{H}, \mathrm{C}_{2}-\mathrm{H}\right), 12.10$ (s, $1 \mathrm{H}, \mathrm{NH}$, $\mathrm{D}_{2} \mathrm{O}$ exchangeable); IR (KBr) $v\left(\mathrm{~cm}^{-1}\right): 3230(\mathrm{NH}), 1700$ $(\mathrm{C}=\mathrm{O}), 1610(\mathrm{NH}-\mathrm{C}=\mathrm{S}), 1550(\mathrm{C}=\mathrm{N})$; MS (EI) $\mathrm{m} / z: 413\left(\mathrm{M}^{+}\right.$, $30 \%), 414\left(\mathrm{M}^{+}+1,8.4 \%\right)$; Anal. Calcd for $\mathrm{C}_{23} \mathrm{H}_{19} \mathrm{~N}_{5} \mathrm{OS}$ 
(413.49): C, 66.81; H, 4.63; N, 16.94; O, 3.87; S, 7.75\%. Found: C, 67.16; H, 4.98; N, 17.31; O, 4.24; S, 8.12\%.

7-Benzyl-2-methyl-5,6-diphenyl-3H-pyrrolo[2,3-d]pyrimidine $-4(7 \mathrm{H})$-thione VIf. Yield: $47 \%$; M.P. $165-167^{\circ} \mathrm{C} ;{ }^{1} \mathrm{H}$ NMR $\left(\mathrm{DMSO}-d_{6}, 300 \mathrm{MHz}\right) \delta(\mathrm{ppm}): 2.29\left(\mathrm{~s}, 3 \mathrm{H}, \mathrm{CH}_{3}\right), 5.78$ $\left(\mathrm{s}, 2 \mathrm{H}, \mathrm{Ph}-\mathrm{CH}_{2}\right), 7.23-7.8\left(\mathrm{~m}, 16 \mathrm{H}, \mathrm{Ar}-\mathrm{H}\right.$ and $\mathrm{NH}, \mathrm{D}_{2} \mathrm{O}$ exchangeable); IR (KBr) $v\left(\mathrm{~cm}^{-1}\right): 3250(\mathrm{NH}), 1605$ (NH$\mathrm{C}=\mathrm{S}), 1570(\mathrm{C}=\mathrm{N})$; MS (EI) m/z: $407\left(\mathrm{M}^{+}, 26 \%\right), 408\left(\mathrm{M}^{+}+\right.$ 1, 3.91\%), $409\left(\mathrm{M}^{+}+2,0.81 \%\right)$; Anal. Calcd for $\mathrm{C}_{26} \mathrm{H}_{21} \mathrm{~N}_{3} \mathrm{~S}$ (407.53): C, 76.63; H, 5.19; N, 10.31; S, 7.87\%. Found: C, 76.56; $\mathrm{H}, 5.11 ; \mathrm{N}, 10.26 ; \mathrm{S}, 7.80 \%$.

2.1.16. General Procedure for the Preparation of Substituted Carbonohydrazonoyl Derivatives VII (Scheme 3). A mixture of I $(0.01 \mathrm{~mol})$ in concentrated $\mathrm{HCl}(10 \mathrm{ml})$ was cooled with stirring to $0-5^{\circ} \mathrm{C}$ under ice, and cooled sodium nitrite solution ( $2.5 \mathrm{~g}$ in $10 \mathrm{~mL}$ of water) was added to it dropwise during 30 minutes. The reaction mixture was then stirred for 30 minutes. Without separation, an ice-cold mixture of active methylene compounds (malononitrile and/or ethyl cyanoacetate) $(0.015 \mathrm{~mol})$ and sodium acetate $(4.10 \mathrm{~g} ; 0.05 \mathrm{~mole})$ in ethanol $(50 \mathrm{~mL})$ were added dropwise with stirring for 15 min. The stirring was continued for 30 minutes under ice and the reaction mixture was then left for $12 \mathrm{~h}$ at room temperature. The precipitate was filtered off and recrystallized from ethanol/ $\mathrm{H}_{2} \mathrm{O}$ to give VII.

(3-Cyano-1-(3,4-dichlorophenyl)-4,5-diphenyl-1H-pyrrol-2-yl) carbon-hydrazonoyl dicyanide VIId. Yield: 56\%; M.P. 102$106^{\circ} \mathrm{C}$; ${ }^{1} \mathrm{H}$ NMR (DMSO-d, $\left.300 \mathrm{MHz}\right) \delta$ (ppm): 6.71 (s, $1 \mathrm{H}$, $\mathrm{NH}$, hydrazone, $\mathrm{D}_{2} \mathrm{O}$ exchangeable), 7.3-7.8 (m, 13H, Ar-H); IR $(\mathrm{KBr}) v\left(\mathrm{~cm}^{-1}\right): 3290(\mathrm{NH}), 2320(\mathrm{C} \equiv \mathrm{N}), 1695(\mathrm{C}=\mathrm{O}), 1585$ $(\mathrm{C}=\mathrm{N})$; MS (EI) $m / z: 481\left(\mathrm{M}^{+}, 19 \%\right), 482\left(\mathrm{M}^{+}+2,12.8 \%\right)$, $483\left(\mathrm{M}^{+}+4,2.3 \%\right)$; Anal. Calcd for $\mathrm{C}_{26} \mathrm{H}_{14} \mathrm{Cl}_{2} \mathrm{~N}_{6}$ (481.33): C, 64.88; H, 2.93; Cl, 14.73; N, 17.46\%. Found: C, 64.67; H, 2.78; Cl, 14.51; N, 17.12\%.

(3-Cyano-1-(1,5-dimethyl-3-oxo-2-phenyl-2,3-dihydro-1Hpyrazol-4-yl)-4-phenyl-1H-pyrrol-2-yl)carbonohydrazonoyl dicyanide VIIe. Yield: 51\%; M.P. $110-114^{\circ} \mathrm{C} ;{ }^{1} \mathrm{H}$ NMR (DMSO$\left.d_{6}, 300 \mathrm{MHz}\right) \delta(\mathrm{ppm}): 2.42\left(\mathrm{~s}, 3 \mathrm{H}, \mathrm{CH}_{3}\right), 3.11\left(\mathrm{~s}, 3 \mathrm{H}, \mathrm{N}-\mathrm{CH}_{3}\right)$, $6.71\left(\mathrm{~s}, 1 \mathrm{H}, \mathrm{NH}\right.$, hydrazone, $\mathrm{D}_{2} \mathrm{O}$ exchangeable), 7.2-7.8 (m, $11 \mathrm{H}, \mathrm{Ar}-\mathrm{H}$, and $\left.\mathrm{C}_{6}-\mathrm{H}\right)$; IR $(\mathrm{KBr}) v\left(\mathrm{~cm}^{-1}\right): 3290(\mathrm{NH}), 2320$ $(\mathrm{C} \equiv \mathrm{N}), 1695(\mathrm{C}=\mathrm{O}), 1585(\mathrm{C}=\mathrm{N})$; MS (EI) $m / z: 446\left(\mathrm{M}^{+}\right.$, 17.8\%), $447\left(\mathrm{M}^{+}+1,8.21 \%\right)$; Anal. Calcd for $\mathrm{C}_{25} \mathrm{H}_{18} \mathrm{~N}_{8} \mathrm{O}$ (446.46): C, 67.25; H, 4.06; N, 25.10; O, 3.58\%. Found: C, $67.54 ; \mathrm{H}, 4.12 ; \mathrm{N}, 25.23 ; \mathrm{O}, 3.69 \%$.

Ethyl 2-(2-(1-Benzyl-3-cyano-4,5-diphenyl-1H-pyrrol-2-yl) hydrazono)-2-cyano-acetate VIIf. Yield: 48\%; M.P. 125$130^{\circ} \mathrm{C} ;{ }^{1} \mathrm{H}$ NMR (DMSO- $\left.d_{6}, 300 \mathrm{MHz}\right) \delta(\mathrm{ppm}): 1.31(\mathrm{t}, 3 \mathrm{H}$, $\left.\mathrm{J}=6.8, \mathrm{CH}_{2}-\mathrm{CH}_{3}^{*}\right), 4.4\left(\mathrm{q}, 2 \mathrm{H}, \mathrm{J}=6.8, \mathrm{O}-\mathrm{CH}_{2}\right), 5.62(\mathrm{~s}, 2 \mathrm{H}$, $\left.\mathrm{Ph}-\mathrm{CH}_{2}\right), 6.8\left(\mathrm{~s}, 1 \mathrm{H}, \mathrm{NH}\right.$, hydrazone, $\mathrm{D}_{2} \mathrm{O}$ exchangeable), 7.2-7.8 (m, 15H, Ar-H, and $\left.\mathrm{C}_{6}-\mathrm{H}\right)$; IR $(\mathrm{KBr}) v\left(\mathrm{~cm}^{-1}\right): 3290$ $(\mathrm{NH}), 2320(\mathrm{C} \equiv \mathrm{N}), 1695(\mathrm{C}=\mathrm{O}), 1585(\mathrm{C}=\mathrm{N})$; MS (EI) $m / z: 473\left(\mathrm{M}^{+}, 18 \%\right), 474\left(\mathrm{M}^{+}+1,5.1 \%\right)$; Anal. Calcd for
$\mathrm{C}_{29} \mathrm{H}_{23} \mathrm{~N}_{5} \mathrm{O}_{2}$ (473.53): C, 73.56; H, 4.90; N, 14.79; O, 6.76\%. Found: C, 73.48; H, 4.64; N, 14.63; O, 6.70\%.

2.1.17. General Procedure for the Preparation of Pyrazolyl Derivatives VIII (Scheme 3). A mixture of compound VII $(0.01 \mathrm{~mol})$ and hydrazine hydrate $(0.64 \mathrm{ml}, 0.02 \mathrm{~mole})$ in ethanol $(30 \mathrm{~mL})$ were heated under reflux for $8 \mathrm{~h}$ controlled by TLC. The solvent was concentrated and the reaction product was allowed to cool then pour on acidified ice $/ \mathrm{H}_{2} \mathrm{O}$. The product was filtered off, washed with water, dried, and recrystallized from ethanol to give VIII.

2-(2-(3,5-Diamino-4H-pyrazol-4-ylidene)hydrazinyl)-1-(3,4dichloro phenyl)-4,5-diphenyl-1H-pyrrole-3-carbonitrile VIIId. Yield: 55\%; M.P. $120-124^{\circ} \mathrm{C}$; ${ }^{1} \mathrm{H}$ NMR (DMSO- $d_{6}$, $300 \mathrm{MHz}) \delta(\mathrm{ppm}): 6.5$ (s, $4 \mathrm{H}, 2^{*} \mathrm{NH}_{2}, \mathrm{D}_{2} \mathrm{O}$ exchangeable), 7.2-8 ( $\mathrm{m}, 14 \mathrm{H}$, Ar- $\mathrm{H}$ and $\mathrm{NH}$, hydrazone, $\mathrm{D}_{2} \mathrm{O}$ exchangeable); IR $(\mathrm{KBr}) v\left(\mathrm{~cm}^{-1}\right)$ : 3350-3280 (broad $\mathrm{NH}$ and $\left.\mathrm{NH}_{2}\right), 2320$ $(\mathrm{C} \equiv \mathrm{N}), 1695(\mathrm{C}=\mathrm{O}), 1585(\mathrm{C}=\mathrm{N})$; MS (EI) $\mathrm{m} / z$ : $513\left(\mathrm{M}^{+}\right.$, $12 \%), 515\left(\mathrm{M}^{+}+2,7.8 \%\right), 516\left(\mathrm{M}^{+}+4,2.4 \%\right)$; Anal. Calcd for $\mathrm{C}_{26} \mathrm{H}_{17} \mathrm{Cl}_{2} \mathrm{~N}_{7} \mathrm{O}$ (513.09): C, 60.71; $\mathrm{H}, 3.33 ; \mathrm{Cl}, 13.79 ; \mathrm{N}$, 19.06; O, 3.11\%. Found: C, 60.98; H, 3.41; Cl, 13.95; N, 19.43; $\mathrm{O}, 3.27 \%$.

2-(2-(3,5-Diamino-4H-pyrazol-4-ylidene)hydrazinyl)-1-(1,5dimethyl-3-oxo-2-phenyl-2,3-dihydro-1H-pyrazol-4-yl)-4phenyl-1H-pyrrole-3-carbonitrile VIIIe. Yield: 61\%; M.P. 135-138 ${ }^{\circ}$; ${ }^{1} \mathrm{H}$ NMR (DMSO- $\left.d_{6}, 300 \mathrm{MHz}\right) \delta$ (ppm): 2.42 $\left(\mathrm{s}, 3 \mathrm{H}, \mathrm{CH}_{3}\right), 3.11\left(\mathrm{~s}, 3 \mathrm{H}, \mathrm{N}-\mathrm{CH}_{3}\right), 6.48\left(\mathrm{~s}, 4 \mathrm{H}, 2^{*} \mathrm{NH}_{2}\right.$, $\mathrm{D}_{2} \mathrm{O}$ exchangeable), $6.89\left(\mathrm{~s}, 1 \mathrm{H}, \mathrm{NH}\right.$, hydrazone, $\mathrm{D}_{2} \mathrm{O}$ exchangeable), 7.3-8 (m, 11H, Ar- $\mathrm{H}$, and $\left.\mathrm{C}_{6}-\mathrm{H}\right)$; IR $(\mathrm{KBr}) v$ $\left(\mathrm{cm}^{-1}\right)$ : 3340-3290 (broad $\mathrm{NH}$ and $\left.\mathrm{NH}_{2}\right), 2320(\mathrm{C} \equiv \mathrm{N}), 1695$ $(\mathrm{C}=\mathrm{O}), 1585(\mathrm{C}=\mathrm{N})$; MS (EI) $m / z$ : $478\left(\mathrm{M}^{+}, 15.2 \%\right), 479\left(\mathrm{M}^{+}\right.$ $+1,4.46 \%$ ); Anal. Calcd for $\mathrm{C}_{25} \mathrm{H}_{22} \mathrm{~N}_{10} \mathrm{O}$ (478.51): C, 62.75; $\mathrm{H}, 4.63$; N, 29.27; O, 3.34\%. Found: C, 62.64; H, 4.47; N, $29.02 ; \mathrm{O}, 3.09 \%$.

2-(2-(3-Amino-5-hydroxy-4H-pyrazol-4-ylidene)hydrazinyl)1-benzyl-4,5-diphenyl-1H-pyrrole-3-carbonitrile VIIIf. Yield: $56 \%$; M.P. $97-100^{\circ} \mathrm{C}$; ${ }^{1} \mathrm{H}$ NMR (DMSO- $\left.d_{6}, 300 \mathrm{MHz}\right)$ $\delta$ (ppm): $5.61\left(\mathrm{~s}, 2 \mathrm{H}, \mathrm{CH}_{2}\right), 6.45\left(\mathrm{~s}, \quad 4 \mathrm{H}, \quad 2^{*} \mathrm{NH}_{2}\right.$, $\mathrm{D}_{2} \mathrm{O}$ exchangeable $), 6.8\left(\mathrm{~s}, 1 \mathrm{H}, \mathrm{NH}\right.$, hydrazone, $\mathrm{D}_{2} \mathrm{O}$ exchangeable), 7.2-7.9 (m, 15H, Ar-H).; IR (KBr) $v\left(\mathrm{~cm}^{-1}\right)$ : 3340-3270 (broad $\mathrm{NH}$ and $\left.\mathrm{NH}_{2}\right), 2310(\mathrm{C} \equiv \mathrm{N}), 1690(\mathrm{C}=\mathrm{O})$, $1575(\mathrm{C}=\mathrm{N})$; $\mathrm{MS}(\mathrm{EI}) \mathrm{m} / z: 459\left(\mathrm{M}^{+}, 10 \%\right), 460\left(\mathrm{M}^{+}+1\right.$, 2.91\%); Anal. Calcd for $\mathrm{C}_{27} \mathrm{H}_{21} \mathrm{~N}_{7} \mathrm{O}$ (459.50): C, 70.57; $\mathrm{H}$, 4.61; N, 21.34; O, 3.48\%. Found: C, 70.65; H, 4.69; N, 21.07; $\mathrm{O}, 3.41 \%$.

\section{Biological Screening}

3.1. Animals. The complete course of the experiment was conducted using male Wistar albino rats (200-250 g), reared and maintained in the animal house of the institution and provided free access to pelleted food and water ad libitum. The rats were maintained in a controlled environment $(12 \mathrm{~h}$ light and dark cycle) for about a week for acclimatization. The protocol of the study was approved by the animal ethics 
committee of the Faculty of Pharmacy, Helwan University (10-01-2012). The study was conducted in accordance with the EC, directive 86/609/EEC for animal experiments.

3.2. Dose Determination. Glimepiride (Amaryl) was used as a standard antidiabetic ( $4 \mathrm{mg} / \mathrm{kg}$ ) in $1 \%$ gum acacia and administered orally [32]. Equivalent doses of all derivatives were calculated according to their molecular weight $[M \cdot w t]$.

3.3. Sucrose-Loaded Model (SLM). Male Wistar rats were fasted overnight. Blood was collected initially and then the compounds were given to corresponding groups consisting of six rats each by oral gavage. A sucrose load of $(10 \mathrm{gm} / \mathrm{kg})$ body weight was given to each rat after half an hour posttest treatment. Blood was collected in 30, 60, 90, and 120 min after sucrose load [33]. The percentages (\%) fallen in blood glucose level were calculated according to the AUC method.

3.4. Toxicity Study. The derivatives, which showed antihyperglycemic activity in this study, were subjected to in vivo acute toxicity study by testing their effect on serum liver and kidney markers.

3.5. Induction of Experimental Diabetes. Diabetes was induced in overnight fasted rats with a single intraperitoneal injection of streptozotocin (STZ) (Sigma-Aldrich, Co., St. Louis, USA. Catalog number: 1001062761) in a dose of $65 \mathrm{mg} / \mathrm{kg}$. STZ was freshly dissolved in ice cold citrate buffer (0.01 M, pH 4.5) prior to injection [34]. After $48 \mathrm{~h}$, rats showing blood glucose level $\geq 200 \mathrm{mg} / \mathrm{dl}$ were included in the experiment [35].

3.6. Experimental Design. Seventy-six rats (fourteen groups of 5-6 rats each) were used to investigate the antihyperglycemic effect of 12 pyrrole and pyrrolo pyrimidine derivatives. Group 1 was diabetic control; Group 2, diabetic + Glimepiride (Amaryl) (4 mg/kg), served as a reference antidiabetic drug. Groups (3-14) were given the various pyrrole derivatives (Ia-e, IVg, VIf, VIIa, b, f, and VIIIf, a, resp.). The treated groups administered the Amaryl and different derivatives orally.

3.7. Methodology. For each group, blood glucose was estimated at zero, one, two, four, and six hours after oral administration of derivatives using glucometer (Gluco Dr Super Sensor, AllMedicus Co., Ltd., Anyang, Gyeonggi, Korea).

Alanine aminotransferase (ALT) and aspartate aminotransferase (AST) activities in serum were measured according to the Reitman-Frankel calorimetric transaminase procedure [36], whereas alkaline phosphatase (ALP) was assayed by the kinetic enzymatic method by measuring the rate of hydrolysis of p-nitrophenyl phosphate by ALP according to Henry [37]; all were measured as indicators of hepatic injury. Serum creatinine levels were assayed as an indicator for renal injury in the samples by a calorimetric method [38], using commercial diagnostic kits (Diamond Diagnostics, Egypt).
3.8. Statistical Analysis. Data were represented as mean area under curve (AUC) \pm SD. Significant differences between groups was tested using GraphPad InStat (Graph software Inc., V 3.05, Ralph Stahlman, Purdue University). Appropriate graphs were plotted using Microsoft Excel 2007. $P$ value less than 0.05 was considered statistically significant.

\section{Results and Discussion}

4.1. Chemistry. The target pyrrole $o$-amino carbonitriles Ia, b, and $\mathbf{d}$ were prepared by the reaction [26-31] of benzoin with appropriate amines and malononitrile in nonpolar solvent. On the other hand, Ic and e were obtained by condensation of $\alpha$-(arylamino)-acetophenone with malononitrile in sodium ethoxide/ethanol.

Compounds Ia-e were utilized for the preparation of pyrrole derivatives If-o using appropriate reagents and reaction conditions; heating Ia-e with triethyl orthoformate (TEOF) afforded the corresponding 2-ethoxy methylamino derivative If-j, while, on react with acetic anhydride, the corresponding 2-acetylamino Ik-o were afforded, as revealed in Scheme 1.

On the other hand, the pyrrole derivatives Ia-e were converted to the corresponding pyrrolo[2,3-d]pyrimidine-4ones IIa-j via condensation with formic acid $[39,40]$ and/or $\mathrm{AcOH} / \mathrm{HCl}[28,41]$, as revealed in Scheme 2.

Interaction [41] of Ia-e with formamide afforded the corresponding 4-amino pyrrolo[2,3-d]pyrimidines IIIa-e, which can also be prepared via stirring of the imidate $\mathbf{I} \mathbf{f}-\mathbf{j}$ with ammonium hydroxide at room temperature, as revealed in Scheme 2. The reaction of pyrrole $o$-amino carbonitriles Ia-e with thiourea in ethanol was reported [42] to afford the corresponding 4-aminopyrimidine-2-thione IIIf-j.

Pyrrolopyrimidinones IIa-j were converted $[41,43,44]$ to its corresponding 4-chloro derivative IVa-j by refluxing with phosphorus oxychloride, as revealed in Scheme 2.

The 4-chloro IVa-j were utilized for the preparation of pyrrolopyrimidine derivatives $\mathbf{V a}-\mathbf{j}$ and $\mathbf{V I a}-\mathbf{j}$ using appropriate reagents and reaction conditions $[40,44]$ : the synthesis of certain 4-hydrazino-7H-pyrrolo[2,3-d]pyrimidines Va-j by hydrazinolysis of the corresponding 4-chloro analogues. Yet, when 4-chloro analogues IVa-j and thiourea were heated [45] in absolute ethanol, the pyrrolopyrimidine$4(3 \mathrm{H})$-thiones VIa-f were obtained, as revealed in Scheme 2. Diazotisation reaction of amino group in 2-amino-pyrrole, followed by coupling of the diazonium salt with active methylenes (ex: malononitrile) has been reported [46-48].

Diazotization of Ia-e using a mixture of sodium nitrite and $\mathrm{HCl}$ (without acetic acid) at $0-5^{\circ} \mathrm{C}$, without separation, adding an active methylene compounds, namely, malononitrile and/or ethyl cyanoacetate in ethanol in the presence of sodium acetate afforded the corresponding hydrazono derivatives VIIa-i. This reaction could be explained via formation of the diazonium chlorides at first, which in addition to malononitrile afforded VIIa-i. Cyclization of hydrazono derivatives 2 using hydrazine hydrate in boiling ethanol leads to the formation of the corresponding pyrazolin-5-one derivatives VIIIa-i, as revealed in Scheme 3. 
TABLE 1: Effect of various treatments on the mean area under curve (AUC) of blood glucose levels in rats.

\begin{tabular}{|c|c|c|}
\hline \multirow[t]{2}{*}{ Tested compound(s) } & \multicolumn{2}{|c|}{$\begin{array}{l}\% \text { reduction in blood glucose compared } \\
\text { to control }\end{array}$} \\
\hline & SLM & STZ \\
\hline Amaryl [Standard drug] & $27.7^{\mathrm{a}}$ & $30.4^{\mathrm{a}}$ \\
\hline$[\mathbf{I a}]$ & $17.4^{\mathrm{a}}$ & $33.3^{\mathrm{a}}$ \\
\hline$[\mathbf{I b}]$ & $10.9^{\mathrm{a}}$ & NA \\
\hline$[\mathbf{I c}]$ & $18^{\mathrm{a}}$ & $35.3^{\mathrm{a}}$ \\
\hline$[\mathbf{I d}]$ & NA & NA \\
\hline$[\mathbf{I e}]$ & $16.7^{\mathrm{a}}$ & $29.5^{\mathrm{a}}$ \\
\hline$[\mathbf{I V g}]$ & $13^{\mathrm{a}}$ & $11.2^{\mathrm{a}}$ \\
\hline$[\mathbf{V I f}]$ & NA & NA \\
\hline [VIIa $]$ & NA & NA \\
\hline [VIIb] & NA & NA \\
\hline [VIIf $]$ & NA & NA \\
\hline [VIIIa] & NA & NA \\
\hline [VIIIf] & NA & NA \\
\hline
\end{tabular}

$\mathrm{NA}=$ not active.

${ }^{a}$ Considered significant compared to control $(P \leq 0.05)$.

SLM: Sucrose-Loaded Model; STZ: Streptozotocin model of diabetes.

4.2. Biological Activities. Twelve of the synthesized Pyrroles and pyrrolopyrimidines were evaluated for their antihyperglycemic activity using both streptozotocin models of diabetes and sucrose load model [32-35]. The synthesized compounds were assessed for their antihyperglycemic activity, which is comparable to Glimepiride (Amaryl) the standard antihyperglycemic drug, by comparing the mean area under the curve (AUC) for the blood glucose level between the different studied groups. The proved pyrrole derivatives, which showed promising decrease in the serum blood glucose level, were subjected to test their toxicity in vivo on serum liver and kidney markers.

The tested compounds were classified into 2 main groups: first, the open form pyrrole derivatives, namely, Ia-e (pyrrole $o$-amino carbonitriles), hydrazone derivatives VIIa, $\mathbf{b}$, and $\mathbf{f}$, and pyrazolin-5-one derivatives VIIIa, f;second, the pyrrolopyrimidines, namely, 4-chloro IVg and 4-thio derivatives VIf.

Only the open form pyrrole derivatives, namely, I a, c, and e (pyrrole $o$-amino carbonitriles), induced a significant decrease in blood glucose level in the sucrose load model $(17.4 \%, 18 \%$, and $16.7 \%$, respectively) compared to the untreated normal control. Moreover, they induced significant decrease in blood glucose level in the STZ model of diabetes $(33.3 \%, 35.3 \%$, and $29.5 \%$, respectively) compared to the diabetic control group, as depicted in Table 1.

Comparing the antihyperglycemic activity of the these compounds with that of the reference antidiabetic drug (Amaryl) showed that compounds Ia, Ic, and Ie showed significant decrease in the blood glucose level $(109.4 \%$, $116.2 \%$, and $97 \%$, respectively) when compared to the activity of Amaryl, as shown in Figure 3.

Among the pyrrolopyrimidines, only the 4-chloro IVg (also bearing the antipyrine moiety at N-pyrrole) showed

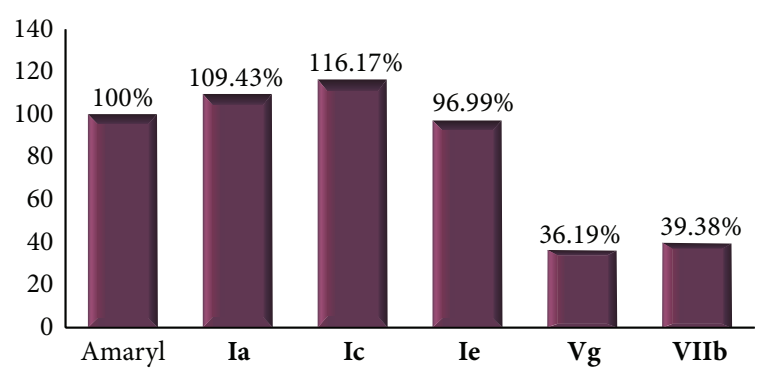

FIGURE 3: Potency of antihyperglycemic derivatives compared to Amaryl.

marked but not significant decrease in blood glucose level $11.2 \%$ compared to the diabetic control group, as shown in Table 1 .

Studying the acute toxicity of the promising antihyperglycemic derivatives Ia, c, and e on the rats showed that the levels of sera ALT, AST, ALP, and creatinine were not significantly changed from that of the control untreated group and, also, the rats did not die or show any toxicity symptoms, as shown in Table 2.

To analyze structure-activity relationships, three structural components were considered: the nature of the heterocycle nucleus, the nature of the side chain of the heterocycle system, and the function of the side chain, as shown in Figure 4.

First, the influence of the nature of the heterocyclic system was easily observed as pyrrole (I a, c, and e) derivatives have show superior activity over pyrrolopyrimidines IVg and VIf.

Regarding the side chain function, for the pyrroles derivatives, the free amino group in pyrrole $o$-amino carbonitriles I a, c, and e conferred the greater activity over the hydrazone derivatives VIIa, $\mathbf{b}$, and $\mathbf{f}$ which showed a marked activity over the pyrazolin-5-one derivatives VIIIa, f, which have no activity. For the pyrrolopyrimidines, the 4-chloro IVg confers markedly but not significantly higher activity than the 4-thio derivatives VIf.

Finally, the influence of the nature of the side chain on the heterocycle system, among the active compounds the antipyrine bearing N7-pyrrole (I e and IVg) showing a good activity over the benzyl (VIf, VIIa and VIIIa).

\section{Conclusion}

In the present study, we described a straightforward and efficient synthesis of some pyrroles and pyrrolo[2,3d]pyrimidine and also,we examined their effects as antihperglycemic agents. The structure-activity relationship (SAR) results indicated that the pyrroles $\mathbf{I a}, \mathbf{c}$, and $\mathbf{e}$ containing amino and cyano groups displayed good to moderate antihyperglycemic activity profile compared to control. On diazotization of the amino group in VII and VIII, this did not enhance the activity. The introduction of chloro group to IVg resulted in an enhanced antihyperglycemic activity of the pyrrolopyrimidine analogs over the hydrazine derivatives. These results and others demonstrated that 
TABLE 2: Effect of compounds Ia, Ic, and Ie on ALT, AST, ALP, and creatinine.

\begin{tabular}{lcccc}
\hline Parameter & Control & Ia & Ic & Ie \\
\hline ALT (U/L) & $22.6 \pm 4.6$ & $17.9 \pm 2.7$ & $27.16 \pm 6.3$ & $20.5 \pm 3.1$ \\
AST (U/L) & $63.4 \pm 14.6$ & $69.4 \pm 9.5$ & $72 \pm 7.8$ & $62.7 \pm 9.7$ \\
ALP (U/L) & $70.6 \pm 15$ & $68.5 \pm 12.3$ & $63.8 \pm 15.4$ & $73.9 \pm 13.2$ \\
Creatinine (mg/dL) & $0.83 \pm 0.13$ & $0.8 \pm 0.16$ & $0.63 \pm 0.09$ & $0.77 \pm 0.14$ \\
\hline
\end{tabular}<smiles>[X]c1c(-c2ccccc2)c(C#N)c(N)n1[Al]</smiles>

\begin{tabular}{lllll}
\multicolumn{1}{c}{$\mathbf{I a}$} & Ib & Ic & Id & Ie \\
$\mathrm{X}=\mathrm{Ph}$ & $\mathrm{Ph}$ & $\mathrm{H}$ & $\mathrm{H}$ & $\mathrm{Ph}$ \\
$\mathrm{Ar}=\mathrm{CH}_{2} \mathrm{Ph}$ & Anti & $(\mathrm{Cl})_{2} \mathrm{Ph}$ & Anti & $(\mathrm{Cl})_{2} \mathrm{Ph}$
\end{tabular}

(a)<smiles>Cc1nc(Cl)c2c(-c3ccccc3)c(-c3ccccc3)n([AlH2])c2n1</smiles>

(b)<smiles>Cc1nc2c(c(-c3ccccc3)c(-c3ccccc3)n2C)c(=S)[nH]1</smiles>

(c)<smiles>[R1]C=NNc1c(C#N)c(-c2ccccc2)c(-c2ccccc2)n1[Al]</smiles>

\begin{tabular}{lll}
$\mathrm{Ar}=\mathrm{CH}_{2} \mathrm{Ph}$ & Anti & \multicolumn{1}{c}{ VIIf } \\
$\mathrm{CH}=\mathrm{CN}$ & $\mathrm{CN}$ & $\mathrm{CN}$ \\
$\mathrm{R}^{\prime}=\mathrm{CN}$ & $\mathrm{CN}$ & COOEt
\end{tabular}

(d)<smiles>N#Cc1cc(-c2ccccc2)n(Cc2ccccc2)c1NC=C1C(N)=NN=C1[Y](O)c1ccccc1</smiles>

(e)

Figure 4: Pyrrole and Pyrrolopyrimidines derivatives; evaluated as antihyperglycemic agents.

the synthesized pyrrole and pyrrolopyrimidine compounds are promising antihyperglycemic agents.

\section{Conflict of Interests}

The authors declare that there is no conflict of interests regarding the publication of this paper.

\section{References}

[1] S. Wang, N. C. Wan, J. Harrison et al., "Design and synthesis of new templates derived from pyrrolopyrimidine as selective multidrug-resistance-associated protein inhibitors in multidrug resistance," Journal of Medicinal Chemistry, vol. 47, no. 6, pp. 1339-1350, 2004.

[2] K. L. Smith, V. C. H. Lai, B. J. Prigaro et al., "Synthesis of new 2' $\beta$-C-methyl related triciribine analogues as anti-HCV agents," Bioorganic \& Medicinal Chemistry Letters, vol. 14, no. 13, pp. 3517-3520, 2004.

[3] T.-C. Chien, E. A. Meade, J. M. Hinkley, and L. B. Townsend, "Facile synthesis of 1-substituted 2-amino-3-cyanopyrroles: new synthetic precursors for 5,6-unsubstituted pyrrolo[2,3d]pyrimidines," Organic Letters, vol. 6, no. 17, pp. 2857-2859, 2004.

[4] A. Budhiraja, K. Kadian, M. Kaur et al., "Synthesis and biological evaluation of naphthalene, furan and pyrrole based chalcones as cytotoxic and antimicrobial agents," Medicinal Chemistry Research, vol. 21, no. 9, pp. 2133-2140, 2012.

[5] M. V. Raimondi, S. Cascioferro, D. Schillaci, and S. Petruso, "Synthesis and antimicrobial activity of new bromine-rich pyrrole derivatives related to monodeoxypyoluteorin," European Journal of Medicinal Chemistry, vol. 41, no. 12, pp. 1439-1445, 2006.

[6] M. Biava, R. Fioravanti, G. C. Porretta, D. Deidda, C. Maullu, and R. Pompei, "New pyrrole derivatives as antimycobacterial agents analogs of BM212," Bioorganic \& Medicinal Chemistry Letters, vol. 9, no. 20, pp. 2983-2988, 1999.

[7] M. Biava, G. C. Porretta, D. Deidda, R. Pompei, A. Tafi, and F. Manetti, "Importance of the thiomorpholine introduction in new pyrrole derivatives as antimycobacterial agents analogues of BM 212," Bioorganic \& Medicinal Chemistry, vol. 11, no. 4, pp. 515-520, 2003.

[8] C. Zhang, J. Dong, T. Cheng, and R. Li, "Y(OTf)3-catalyzed novel Mannich reaction of $N$-alkoxycarbonylpyrroles, formaldehyde and primary amine hydrochlorides," Tetrahedron Letters, vol. 42, no. 3, pp. 461-463, 2001.

[9] U. Uršič, J. Svete, and B. Stanovnik, "Synthesis of 4-(2-hydroxy1-methyl-5-oxo- $1 H$-imidazol-4(5H)-ylidene)-5-oxo-1-aryl4,5-dihydro- $1 H$-pyrrole-3-carboxylates, a new triazafulvalene system," Tetrahedron, vol. 66, no. 24, pp. 4346-4356, 2010.

[10] M. C. Rodríguez-Argüelles, E. C. López-Silva, J. Sanmartín, P. Pelagatti, and F. Zani, "Copper complexes of imidazole2-, pyrrole-2- and indol-3-carbaldehyde thiosemicarbazones: 
inhibitory activity against fungi and bacteria," Journal of Inorganic Biochemistry, vol. 99, no. 11, pp. 2231-2239, 2005.

[11] F. Micheli, R. Di Fabio, F. Bordi et al., “2,4-Dicarboxy-pyrroles as selective non-competitive mGluR1 antagonists: further characterization of 3,5-dimethyl pyrrole-2,4-dicarboxylic acid 2propyl ester 4-(1,2,2-trimethyl-propyl) ester and structureactivity relationships," Bioorganic \& Medicinal Chemistry Letters, vol. 13, no. 13, pp. 2113-2118, 2003.

[12] E. Fernandes, D. Costa, S. A. Toste, J. L. F. C. Lima, and S. Reis, "In vitro scavenging activity for reactive oxygen and nitrogen species by nonsteroidal anti-inflammatory indole, pyrrole, and oxazole derivative drugs," Free Radical Biology \& Medicine, vol. 37, no. 11, pp. 1895-1905, 2004.

[13] S. B. Etcheverry, D. A. Barrio, A. M. Cortizo, and P. A. M. Williams, "Three new vanadyl(IV) complexes with nonsteroidal anti-inflammatory drugs (Ibuprofen, Naproxen and Tolmetin): bioactivity on osteoblast-like cells in culture," Journal of Inorganic Biochemistry, vol. 88, no. 1, pp. 94-100, 2002.

[14] I. Lessigiarska, A. Nankov, A. Bocheva, I. Pajeva, and A. Bijev, "3D-QSAR and preliminary evaluation of anti-inflammatory activity of series of $N$-pyrrolylcarboxylic acids," Il Farmaco, vol. 60, no. 3, pp. 209-218, 2005.

[15] A. Mai, S. Valente, D. Rotili et al., "Novel pyrrole-containing histone deacetylase inhibitors endowed with cytodifferentiation activity," International Journal of Biochemistry and Cell Biology, vol. 39, no. 7-8, pp. 1510-1522, 2007.

[16] M. Sechi, A. Mura, L. Sannia, M. Orecchioni, and G. Paglietti, "Synthesis of pyrrolo[1,2-a]indole-1,8(5H)-diones as new synthons for developing novel tryciclic compounds of pharmaceutical interest," Arkivoc, vol. 2004, no. 5, pp. 97-106, 2004.

[17] Lipitor, LAB-0021-7. 0, Pfizer Ireland Pharmaceuticals, 2004.

[18] R. N. Madadi, N. R. Penthala, V. Janganati, and P. A. Crooks, "Synthesis and anti-proliferative activity of aromatic substituted 5-((1-benzyl-1H-indol-3-yl)methylene)-1,3dimethylpyrimidine-2, 4, 6(1H,3H,5H)-trione analogs against human tumor cell lines," Bioorganic \& Medicinal Chemistry Letters, vol. 24, no. 2, pp. 601-603, 2014.

[19] T. Hirose, H. Asakawa, Y. Itoh et al., "Efficacy of glimepiride in type 2 diabetic patients treated with glibenclamide," Diabetes Research and Clinical Practice, vol. 66, supplement 1, pp. S129S132, 2004.

[20] A. Goel, N. Agarwal, F. V. Singh et al., "Antihyperglycemic activity of 2-methyl-3,4,5-triaryl-1H-pyrroles in SLM and STZ models," Bioorganic \& Medicinal Chemistry Letters, vol. 14, no. 5, pp. 1089-1092, 2004.

[21] H. Xie, L. Zeng, S. Zeng et al., "Novel pyrrolopyrimidine analogues as potent dipeptidyl peptidase IV inhibitors based on pharmacokinetic property-driven optimization," European Journal of Medicinal Chemistry, vol. 52, pp. 205-212, 2012.

[22] S. Sancheti, S. Sancheti, and S.-Y. Seo, "Chaenomeles sinensis: a potent $\alpha$-and $\beta$-glucosidase inhibitor," The American Journal of Pharmacology and Toxicology, vol. 4, no. 1, pp. 8-11, 2009.

[23] J. Deng, L. Peng, G. Zhang et al., "The highly potent and selective dipeptidyl peptidase IV inhibitors bearing a thienopyrimidine scaffold effectively treat type 2 diabetes," European Journal of Medicinal Chemistry, vol. 46, no. 1, pp. 71-76, 2011.

[24] D. J. Augeri, J. A. Robl, D. A. Betebenner et al., "Discovery and preclinical profile of saxagliptin (BMS-477118): a highly potent, long-acting, orally active dipeptidyl peptidase IV inhibitor for the treatment of type 2 diabetes," Journal of Medicinal Chemistry, vol. 48, no. 15, pp. 5025-5037, 2005.
[25] G. Zhao, C. Kwon, A. Wang et al., "Substituted piperidinyl glycinyl 2-cyano-4,5-methano pyrrolidines as potent and stable dipeptidyl peptidase IV inhibitors," Bioorganic \& Medicinal Chemistry Letters, vol. 23, no. 6, pp. 1622-1625, 2013.

[26] M. S. Mohamed, R. Kamel, and S. S. Fatahala, "Synthesis and biological evaluation of some thio containing pyrrolo [2,3d] pyrimidine derivatives for their anti-inflammator and antimicrobial activities," European Journal of Medicinal Chemistry, vol. 45, no. 7, pp. 2994-3004, 2010.

[27] A. E. Rashad, M. S. Mohamed, M. E. A. Zaki, and S. S. Fatahala, "Synthesis and biological evaluation of some pyrrolo[2,3d]pyrimidines," Archiv der Pharmazie, vol. 339, no. 12, pp. 664669, 2006.

[28] M. S. Mohamed, R. Kamel, and S. S. Fathallah, "Synthesis of new pyrroles of potential anti-inflammatory activity," Archiv der Pharmazie, vol. 344, no. 12, pp. 830-839, 2011.

[29] M. S. Mohamed, R. Kamel, and S. S. Fatahala, "New condensed pyrroles of potential biological interest: syntheses and structure-activity relationship studies," European Journal of Medicinal Chemistry, vol. 46, no. 7, pp. 3022-3029, 2011.

[30] M. S. Mohamed and S. S. Fatahala, "Pyrroles and fused pyrroles: synthesis and therapeutic activities," in Mini-Reviews in Organic Chemistry, 2014.

[31] P. M. Traxler, P. Furet, H. Mett, E. Buchdunger, T. Meyer, and N. Lydon, "4-(Phenylamino)pyrrolopyrimidines: potent and selective, ATP site directed inhibitors of the EGF-receptor protein tyrosine kinase," Journal of Medicinal Chemistry, vol. 39, no. 12, pp. 2285-2292, 1996.

[32] A. Mowla, M. Alauddin, M. A. Rahman, and K. Ahmed, "Antihyperglycemic effect of Trigonella foenum-graecum (fenugreek) seed extract in alloxan-induced diabetic rats and its use in diabetes mellitus: A brief qualitative phytochemical and acute toxicity test on the extract," African Journal of Traditional, Complementary and Alternative Medicines, vol. 6, no. 3, pp. 255261, 2009.

[33] F. V. Singh, A. Parihar, S. Chaurasia et al., "5,6-Diarylanthranilo1,3-dinitriles as a new class of antihyperglycemic agents," Bioorganic \& Medicinal Chemistry Letters, vol. 19, no. 8, pp. 2158-2161, 2009.

[34] G. D’Andrea, A. R. Lizzi, F. Brisdelli, A. M. D’Alessandro, A. Bozzi, and A. Oratore, "Protein glycans alteration and a differentdistribution of some enzymatic activities involvedin the glycan processing are found in AZT-treated K562 cells," Molecular and Cellular Biochemistry, vol. 252, no. 1-2, pp. 4551, 2003.

[35] N. N. Kim, M. Stankovic, T. T. Cushman, I. Goldstein, R. Munarriz, and A. M. Traish, "Streptozotocin-induced diabetes in the rat is associated with changes in vaginal hemodynamics, morphology and biochemical markers," BMC Physiology, vol. 6, article 4, 2006.

[36] S. Reitman and S. A. Frankel, "A colorimetric method for the determination of serum glutamic oxalacetic and glutamic pyruvic transaminases," The American Journal of Clinical Pathology, vol. 28, no. 1, pp. 56-63, 1957.

[37] R. J. Henry, Clinical Chemistry: Principles and Techniques, Harper \& Row, New York, NY, USA, 1964.

[38] G. Szasz, U. Boerner, E. W. Busch, and W. Bablok, "Enzymatic assay of creatinine in serum: comparison with Jaffe methods," Journal of Clinical Chemistry and Clinical Biochemistry, vol. 17, no. 11, pp. 683-687, 1979. 
[39] C. G. Dave and N. D. Desai, "Synthesis and reactions of fluoroaryl substituted 2-amino-3-cyanopyrroles and pyrrolo[2,3d] pyrimidines," Journal of Heterocyclic Chemistry, vol. 36, no. 3, pp. 729-733, 1999.

[40] M. M. Ghorab, F. A. Ragab, H. I. Heiba, H. A. Youssef, and M. G. El-Gazzar, "Synthesis of novel pyrrole and pyrrolo[2,3d]pyrimidine derivatives bearing sulfonamide moiety for evaluation as anticancer and radiosensitizing agents," Bioorganic and Medicinal Chemistry Letters, vol. 20, no. 21, pp. 6316-6320, 2010.

[41] B. S. Holla, M. Mahalinga, M. S. Karthikeyan, P. M. Akberali, and N. S. Shetty, "Synthesis of some novel pyrazolo[3,4d]pyrimidine derivatives as potential antimicrobial agents," Bioorganic \& Medicinal Chemistry, vol. 14, no. 6, pp. 2040-2047, 2006.

[42] M. S. A. El-Gaby, A. M. Gaber, A. A. Atalla, and K. A. Abd AlWahab, "Novel synthesis and antifungal activity of pyrrole and pyrrolo[2,3- $d]$ pyrimidine derivatives containing sulfonamido moieties," Il Farmaco, vol. 57, no. 8, pp. 613-617, 2002.

[43] C. G. Dave and R. D. Shah, "Synthesis of $7 H$-tetrazolo[1,5c]pyrrolo[3,2-e]pyrimidines and their reductive ring cleavage to 4-aminopyrrolo[2,3-d]pyrimidines," Journal of Heterocyclic Chemistry, vol. 35, no. 6, pp. 1295-1300, 1998.

[44] A. M. El-Ghanam, "Synthesis of some new spirothiopyran derivatives from the reaction of 4-thiopyrylidenemalononitriles with bidentate and active methylene reagents," Phosphorus, Sulfur and Silicon and the Related Elements, vol. 178, no. 4, pp. 863-868, 2003.

[45] A. Lauria, P. Diana, P. Barraja et al., "New tricyclic systems of biological interest: annelated 1,2,3-triazolo[1,5-a]pyrimidines through domino reaction of 3-azidopyrroles and methylene active nitriles," Tetrahedron, vol. 58, no. 48, pp. 9723-9727, 2002.

[46] A. Lauria, P. Diana, P. Barraja et al., "Pyrrolo[2,1d] $[1,2,3,5]$ tetrazine- $4(3 H)$-ones, a new class of azolotetrazines with potent antitumor activity," Bioorganic \& Medicinal Chemistry, vol. 11, no. 11, pp. 2371-2380, 2003.

[47] M. A. Khan and A. C. C. Freitas, "Fused pyrazolopyrimidines. I. Pyrazolo[4, 3-e]-v-triazolo[1, 5-a]pyrimidine: a new heterocyclic system," Journal of Heterocyclic Chemistry, vol. 17, no. 7, pp. 1603-1604, 1980.

[48] A. P. Freitas, M. Fernanda, J. R. P. Proença, and B. L. Booth, "Synthesis of 5-azido-4-cyanoimidazole and its reaction with active methylene compounds," Journal of Heterocyclic Chemistry, vol. 32, no. 2, pp. 457-462, 1995. 

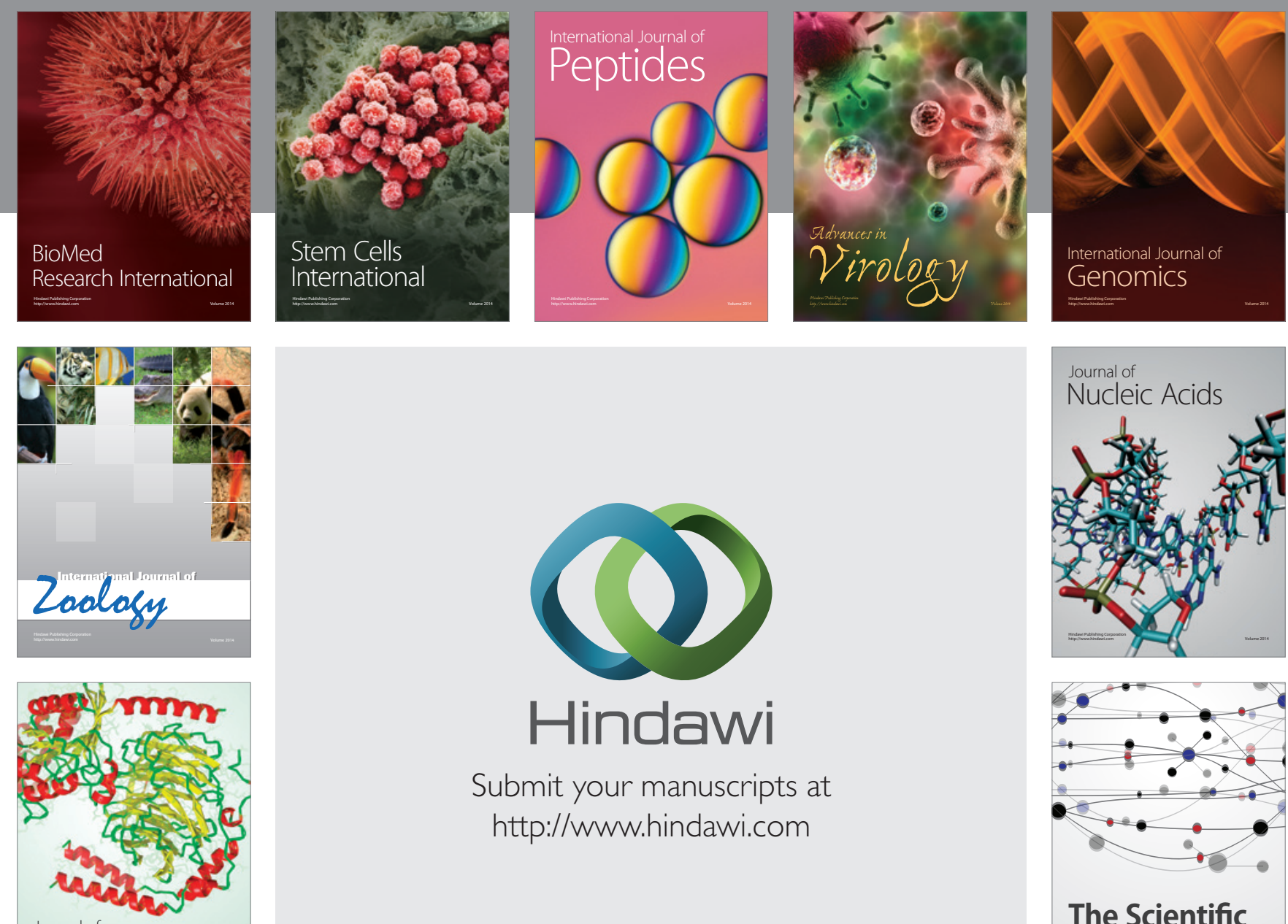

Submit your manuscripts at

http://www.hindawi.com

Journal of
Signal Transduction
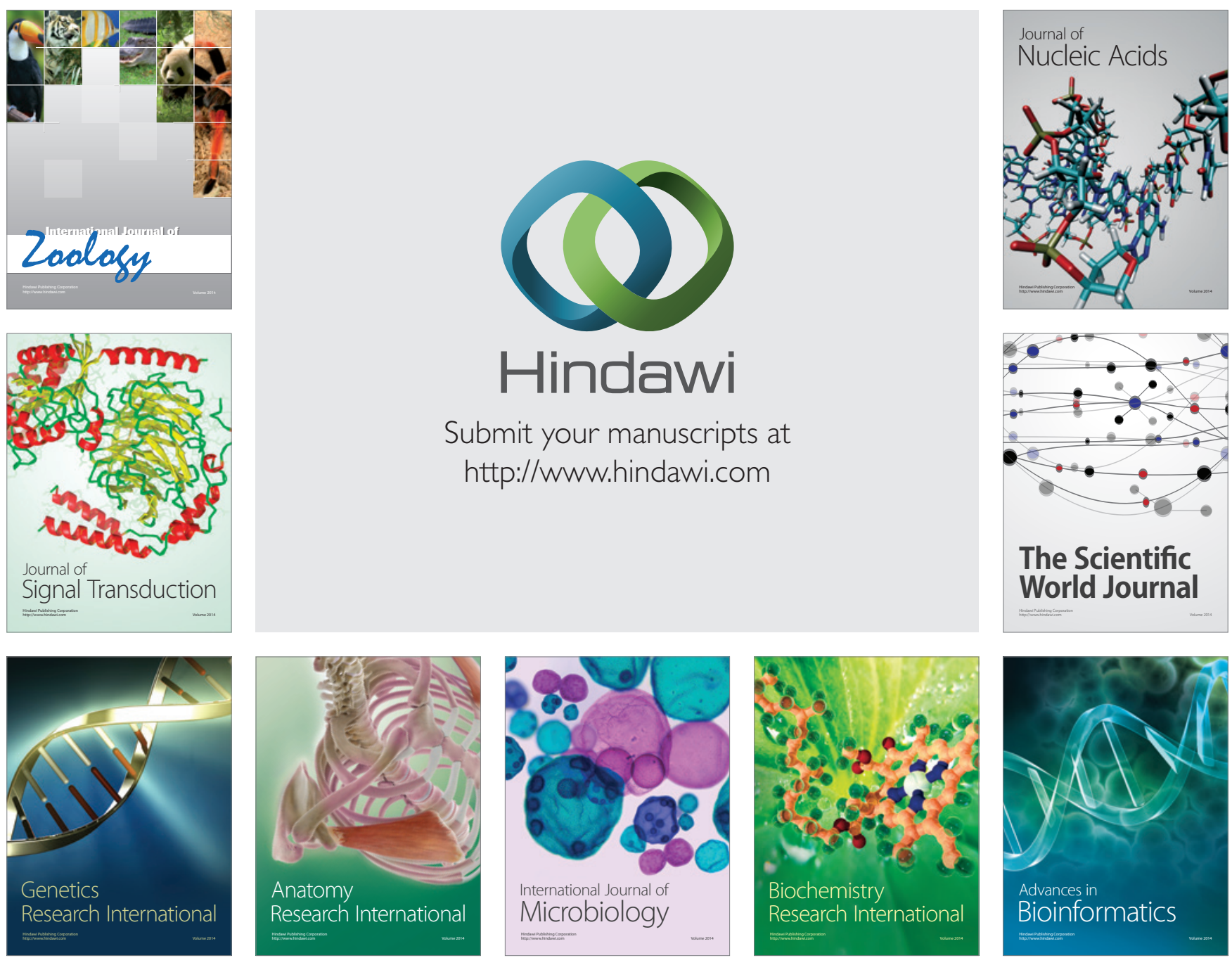

The Scientific World Journal
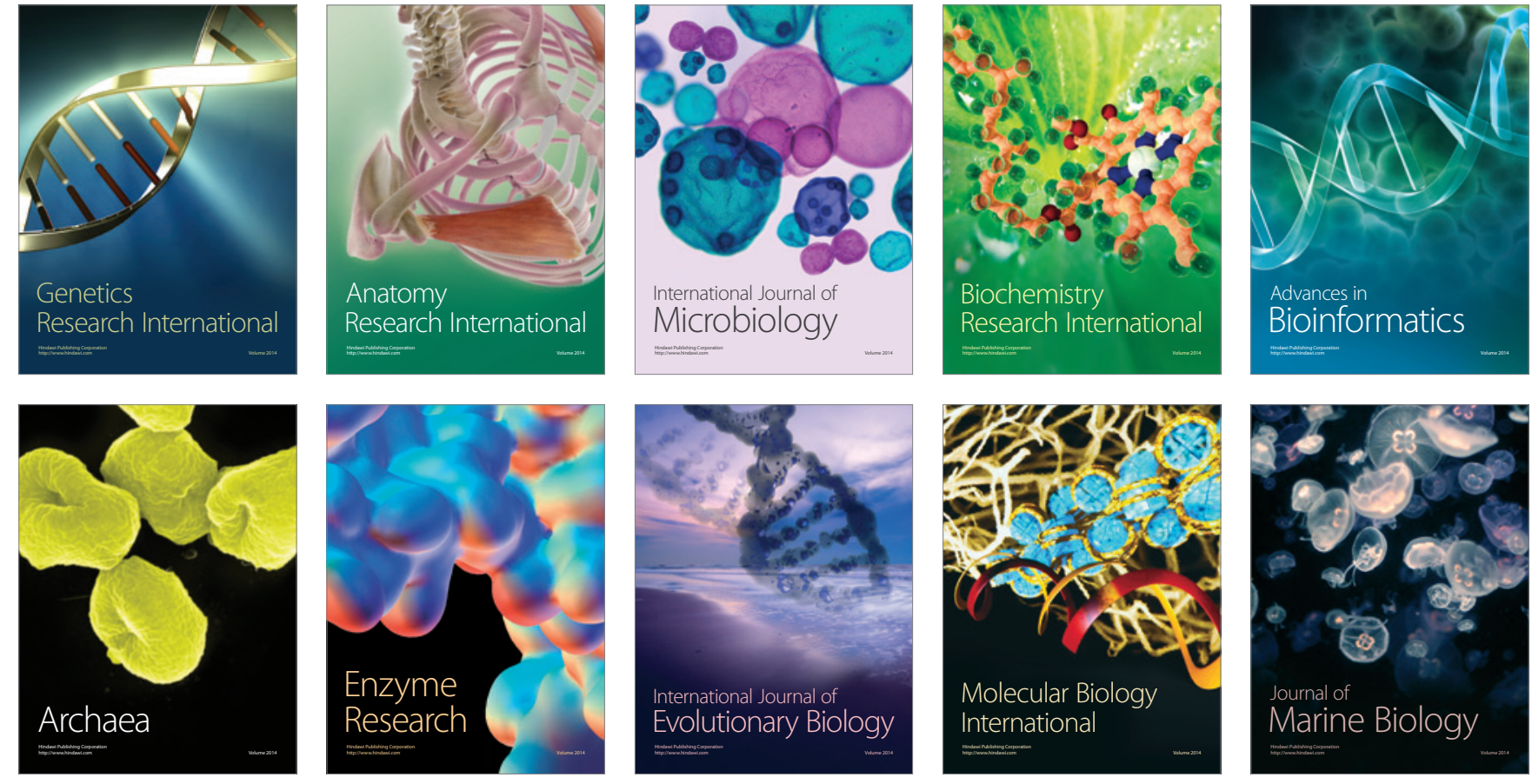